\title{
"Spatial heterogeneity of environmental risk in randomized prevention trials: consequences and modeling"
}

\author{
Abdoulaye Guindo ${ }^{1,2^{*}}$ (D, Issaka Sagara ${ }^{1,2}$, Boukary Ouedraogo 1,3, Kankoe Sallah 1,4, Mahamadoun Hamady Assadou²,
} Sara Healy ${ }^{5}$, Patrick Duffy ${ }^{5}$, Ogobara K. Doumbo ${ }^{1,2^{\wedge}}$, Alassane Dicko ${ }^{2}$, Roch Giorgi ${ }^{6}$ and Jean Gaudart ${ }^{6}$

\begin{abstract}
Background: In the context of environmentally influenced communicable diseases, proximity to environmental sources results in spatial heterogeneity of risk, which is sometimes difficult to measure in the field. Most prevention trials use randomization to achieve comparability between groups, thus failing to account for heterogeneity. This study aimed to determine under what conditions spatial heterogeneity biases the results of randomized prevention trials, and to compare different approaches to modeling this heterogeneity.

Methods: Using the example of a malaria prevention trial, simulations were performed to quantify the impact of spatial heterogeneity and to compare different models.

Simulated scenarios combined variation in baseline risk, a continuous protective factor (age), a non-related factor (sex), and a binary protective factor (preventive treatment). Simulated spatial heterogeneity scenarios combined variation in breeding site density and effect, location, and population density.

The performances of the following five statistical models were assessed: a non-spatial Cox Proportional Hazard (Cox-PH) model and four models accounting for spatial heterogeneity-i.e., a Data-Generating Model, a Generalized Additive Model (GAM), and two Stochastic Partial Differential Equation (SPDE) models, one modeling survival time and the other the number of events. Using a Bayesian approach, we estimated the SPDE models with an Integrated Nested Laplace Approximation algorithm.

For each factor (age, sex, treatment), model performances were assessed by quantifying parameter estimation biases, mean square errors, confidence interval coverage rates (CRs), and significance rates. The four models were applied to data from a malaria transmission blocking vaccine candidate.

Results: The level of baseline risk did not affect our estimates. However, with a high breeding site density and a strong breeding site effect, the Cox-PH and GAM models underestimated the age and treatment effects (but not the sex effect) with a low CR.

When population density was low, the Cox-SPDE model slightly overestimated the effect of related factors (age, treatment). The two SPDE models corrected the impact of spatial heterogeneity, thus providing the best estimates.

(Continued on next page)
\end{abstract}

\footnotetext{
* Correspondence: abdoulaye.guindo@etu.univ-amu.fr

Deceased

${ }^{1}$ Aix Marseille Univ, INSERM, IRD, SESSTIM, Sciences Economiques \& Sociales

de la Santé \& Traitement de l'Information Médicale, Marseille, France

${ }^{2}$ Malaria Research and Training Center - Ogobara K Doumbo, FMOS-FAPH,

Mali-NIAID-ICER, Université des Sciences, des Techniques et des Technologies

de Bamako, Bamako, Mali

Full list of author information is available at the end of the article
}

(c) The Author(s). 2019 Open Access This article is distributed under the terms of the Creative Commons Attribution 4.0 International License (http://creativecommons.org/licenses/by/4.0/), which permits unrestricted use, distribution, and reproduction in any medium, provided you give appropriate credit to the original author(s) and the source, provide a link to the Creative Commons license, and indicate if changes were made. The Creative Commons Public Domain Dedication waiver (http://creativecommons.org/publicdomain/zero/1.0/) applies to the data made available in this article, unless otherwise stated. 
(Continued from previous page)

Conclusion: Our results show that when spatial heterogeneity is important but not measured, randomization alone cannot achieve comparability between groups. In such cases, prevention trials should model spatial heterogeneity with an adapted method.

Trial registration: The dataset used for the application example was extracted from Vaccine Trial \#NCT02334462 (ClinicalTrials.gov registry).

Keywords: Randomized prevention trials, Spatial heterogeneity, Stochastic Partial Differential Equation, Integrated Nested Laplace Approximation, Environmental factors

\section{Background}

In the context of communicable diseases such as vectorborne diseases (e.g., malaria, dengue) or other environmentally influenced diseases (e.g., cholera), the location of individuals affects environmental risk. In the case of vector-borne diseases, proximity to an environment favorable to disease transmission (a vector breeding site or an area favorable to mosquito survival) leads to spatial heterogeneity of risk [1]. Most researchers conducting prevention trials consider that randomization is sufficient to achieve comparability between groups, even when they fail to measure environmental risk. Such prevention trials are based on analysis plans that generally fail to account for spatial heterogeneity [2-5]. In some cases, they account for this heterogeneity by using mixed models [6-8], which associate a random spatial effect with a specific spatial scale (country, region, health district, village, etc.), on the assumption that environmental risk is homogeneous on the scale considered and that no spatial interaction occurs between scales (interscale spatial independence). However, spatial heterogeneity of incidence exists on the village scale itself [9], undermining the conditions for applying such models.

Spatial heterogeneity of risk is often difficult to assess. In contexts where disease risk is associated with environmental factors, specific risk factors are not always measurable or can be measured only through in-depth fieldwork. For example, mosquito breeding sites can be small, numerous, and scattered, as is the case for Anopheles sp. and Aedes sp. (vectors for malaria and dengue, respectively) [10-14]. Similarly, in the context of cholera, it is almost impossible to investigate all water sources (wells or pumps).

While the Cox Proportional Hazard (Cox-PH) model is the most widely used multivariate approach in clinical trials, it is not always applied correctly in contexts of spatial heterogeneity. In view of this, different methods have been proposed that take into account spatial heterogeneity for survival analysis. Generalized Additive Models (GAMs), which were initially developed to model non-linear relationships, are used more and more to model spatial heterogeneity using bivariate spline functions on geographical coordinates as covariates [15-
17]. Alternatively, Stochastic Partial Differential Equation (SPDE) models are used to model explicitly outcome variations following the first law of geography [18]. Thus, Lindgren et al. have proposed an SPDE model solved using a Gaussian field with a Matèrn covariance function that has good computational properties [19, 20]. Moreover, the SPDE model implemented using the INLA (Integreted Nested Laplace Approximation) algorithm is now used in an ever-wider range of contexts [21-23].

The aim of this study was first to determine under what conditions and to what extent spatial heterogeneity of environmental risk can bias the results of randomized prevention trials, and then to compare the performance of different spatial models in estimating treatment effectiveness. These methods were applied to a malaria vaccine trial conducted in Mali.

\section{Methods}

We simulated 432 scenarios (50 datasets for each scenario, 1,000 individuals for each dataset). These scenarios were analyzed first with a non-spatial Cox-PH model, and then with four models that accounted for spatial heterogeneity, including a Data-Generating model (DGM).

For each factor (age, sex, and treatment), model performance was assessed by quantifying parameter estimation bias, mean square error (MSE), confidence interval coverage rate (CR), and significance rate (SR).

For the main scenarios (32), 500 datasets of 1,000 individuals each were performed for validation (Additional file 1: Figure S1).

\section{Simulation plan}

Event time was simulated using a classic Cox-PH model that accounted for different risks factors (including environmental factors) according to a Weibull distribution. Censoring time was simulated using an exponential distribution.

More precisely, for a given vector of covariates $X$, the instantaneous risk function $\lambda_{i}(t, X)$ for individual $i$ at time $t$ was defined as follows: 


$$
\lambda_{i}(t, X)=\lambda_{i}\left(t, X^{(i)}\right)+\lambda_{i}\left(t, X^{(-i)}\right)
$$

where $\lambda_{i}\left(t, X^{(i)}\right)$ was the fixed effect dependent on individual $i$ and $\lambda_{i}\left(t, X^{(-i)}\right)$ was the spatial effect dependent on the neighborhood of individual $i$.

We then simulated a randomized and controlled prevention trial of $n=1,000$ individuals in a square area $\Omega$ of $400 \mathrm{~km}^{2}$. Before the treatment randomization was simulated, different risk situations (scenarios) were simulated according to location (spatial distribution of individuals), baseline risk, and heterogeneity of environmental risk (location and density of breeding sites) (see description below). In addition to the treatment effect, two nonspatial risk factors were simulated: one had a significant continuous effect (age factor), and the other had a null effect (sex factor).

The size of datasets and the number of simulations were selected with an accuracy of 0.01 and a variance of 0.09 , following standard recommendations [24].

\section{Location (spatial distribution of individuals)}

To simulate the spatial distribution of individuals in the study zone $\Omega$, we used an Inhomogeneous Point Process (IPP) in which population density depended on the location of individuals. We considered three clusters, $L 1, L 2$, and $L 3$, where the population was at its densest, and we introduced a concentration parameter $\tau$ to control for population density in these clusters.

The location of these three clusters was randomized using a Homogeneous Point Process (HPP) so as to respect a minimum distance between them (see Additional file 2).

We studied the impact of population density in the three clusters by simulating three different density situations:

$\tau=0.2$ (low population density in the three clusters)

$\tau=0.5$ (average population density in the three clusters)

$\tau=0.8$ (high population density in the three clusters)

\section{Baseline risk}

Baseline risk $\lambda_{0}$ was assumed to be constant for all individuals. It was simulated using a Weibull distribution with a shape parameter set to 1 to obtain a constant value over time. Three baseline risk levels corresponding to three observed epidemiological profiles were used for the scale parameter $\gamma$, taking as an example malaria prevalence in Mali [25]:

Low prevalence: $\gamma=6 \%$ (e.g., situation in Bamako), Median prevalence: $\gamma=37 \%$ (e.g., situation in Segou), High prevalence: $\gamma=60 \%$ (e.g., situation in Mopti).

\section{Source of environmental risk (Breeding sites)}

Spatial heterogeneity of environmental risk was simulated according to location of breeding site, variation in breeding site effect (defined as Relative Risk $R R_{b}$ ), variation in breeding site density, and influence radius of breeding site considered as a constant.

An individual was considered exposed to a breeding site when the distance between this individual and the center of the site was less than the site's influence radius.

The influence radius of a breeding site was interpreted as the average distance traveled by a mosquito to take a blood meal. The influence radius was set at $r=600 \mathrm{~m}$ [26], and was constant for all breeding sites.

Considering that breeding site density did not depend on location, we simulated the spatial distribution of breeding sites following a marked HPP where the mark was the breeding site's influence radius $r$. Thus, an individual could be exposed to zero, one, or more breeding sites, with risk increasing accordingly.

Breeding site density was used to quantify the ratio of the environmental risk area (i.e., the area covered by the influence radius of at least one breeding site) to the total area of the study zone $\left(400 \mathrm{~km}^{2}\right)$.

This density was interpreted as the probability of being exposed to at least one breeding site (see Additional file 2 ). To simulate different scenarios, breeding site density was set at $0.25,0.5$, and 0.75 .

Given that breeding site productivity and protection against mosquito bites can be highly variable, the breeding site effect (defined as Relative Risk $R R_{b}$ ), was also simulated in four situations:

Very weak effect $\left(R R_{b}=1.05\right)$,

Weak effect $\left(R R_{b}=1.20\right)$,

Strong effect $\left(R R_{b}=1.5\right)$,

Very strong effect $\left(R R_{b}=3\right)$.

\section{Treatment}

Allocation to control and intervention groups was simulated according to a symmetric Bernoulli distribution $\mathcal{B}($ $n, 0.5)$ using individual randomization and without accounting for location or for other factors.

Subsequently, four levels of treatment effect (defined as Relative Risk $R R_{t}$ ) were simulated:

Very weak effect $\left(R R_{t}=0.95\right)$,

Weak effect $\left(R R_{t}=0.8\right)$,

Strong effect $\left(R R_{t}=0.6\right)$,

Very strong effect $\left(R R_{t}=0.25\right)$.

Indeed, while the effectiveness of prevention trials (including malaria-prevention trials) is highly variable, in 


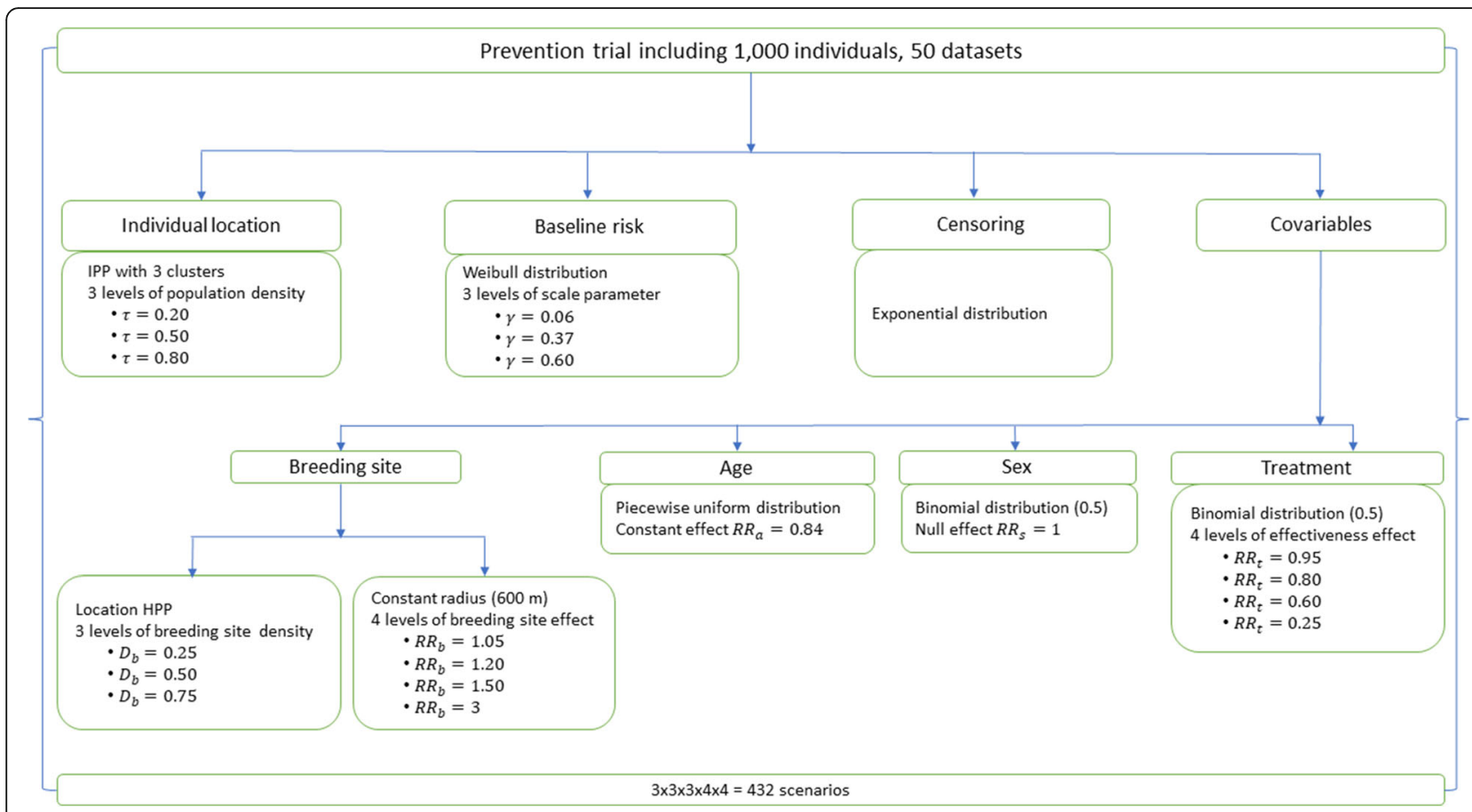

Fig. 1 Simulation scheme for the different scenarios

most cases it falls within the relative risk range of 0.25 to 0.95 [27-32].

\section{Other risk factors}

Although the aim of our study was to assess the impact of spatial heterogeneity of environmental risk, we included two additional factors in our analysis: one was related to the disease (age), and the other was not (sex). These two factors were independent of spatial location, and their effect was constant over time.

Thus, a binary variable identified as the sex factor was simulated using a symmetrical binomial distribution with a null effect (defined as Relative Risk $R R_{s}=1$ ).

$$
\operatorname{Sex} \sim \mathcal{B}(n, p=0.5)
$$

A continuous disease-related variable identified as the age factor was simulated following a piecewise uniform distribution, based on the population distribution by age group in Mali [33]. The protective effect of this variable (defined as Relative Risk $R R_{a}$ ), which was taken from the literature [25], was fixed and constant over time $\left(R R_{a}=\right.$ $0.84)$ :

$$
\begin{aligned}
& \text { Age1 } \sim \mathcal{U}(47.3 \% \mathrm{n},[0.25,14]) \\
& \text { Age2 } \sim \mathcal{U}(19.2 \% \mathrm{n},] 14,24]) \\
& \text { Age3 } \sim \mathcal{U}(26.8 \% \mathrm{n},] 24,54]) \\
& \text { Age4 } \sim \mathcal{U}(3.76 \% \mathrm{n},] 54,64])
\end{aligned}
$$

$$
\text { Age5 } \sim \mathcal{U}(2.94 \% \mathrm{n},] 64,75])
$$

\section{Events and Censoring}

Event time $T_{\text {event }}$ was simulated using a classic Cox-PH model that accounted for the different risk factors detailed above, namely baseline risk, age, sex, treatment, and environmental risk (breeding sites). The instantaneous risk function of individual $i$ was calculated as follows:

$\lambda_{i}(t \mid X)=\lambda_{0} \exp \left(\beta_{1}\right.$ Age $_{i}+\beta_{2}$ Sex $_{i}+\beta_{3}$ Treatment $\left._{i}+\beta_{4} B S_{i}\right)$ [equation 1]

$\lambda_{0}$ was the baseline risk considered constant over time, $B S_{i}$ was the number of breeding sites to which individual $i$ was exposed, and $\beta_{1}, \beta_{2}, \beta_{3}, \beta_{4}$ were the parameters associated with the covariates age, sex, treatment, and breeding site, respectively.

Censoring time $T_{\text {cens }}$ was simulated following an exponential distribution. Thus, observation time $T$ was defined as the minimum between censoring time and event time.

$$
T=\min \left(T_{\text {event }}, T_{\text {cens }}\right)
$$

The combination of these different simulation parameters resulted in 432 scenarios, each containing 50 datasets (Figure 1). Datasets of 1,000 individuals each yielded a power greater than $85 \%$ when the treatment effect $\left(R R_{t}\right)$ was less than 0.8 . For a treatment effect set at $R R_{t}=0.95$, power was around $65 \%$. 


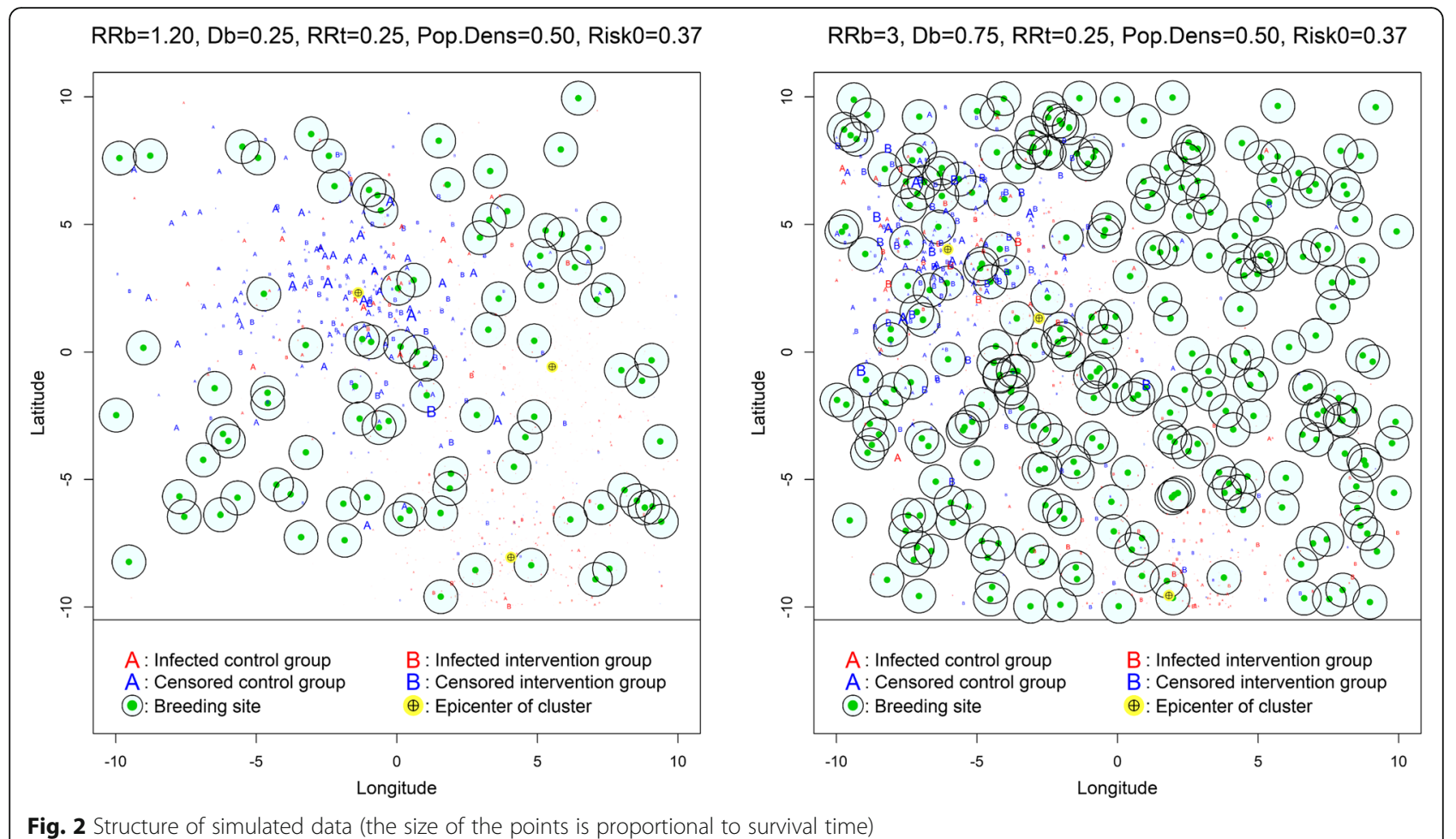

An example of the simulated data structure is shown in Figure 2.

\section{Model Evaluation and Comparison}

For each scenario, we analyzed each dataset using five statistical models (see Additional file 1 for details):

a DGM, according to equation 1;

Model Evaluation and Comparisona classic non-spatial Cox-PH model, whose parameters were estimated with the maximum likelihood method, according to an equation similar to equation 1 but without taking into account the environmental risk factor [34, 35];

a GAM model, also with proportional hazards, which modeled the spatial effect with a bivariate spline function [36] (as detailed below);

two SPDE models (as detailed below), which modeled spatial heterogeneity using a Gaussian field with a Matèrn covariance function: one modeled survival time with a Weibull distribution (Cox-SPDE), and the other modeled the number of events with a Poisson distribution (P-SPDE).

Using a Bayesian approach, we estimated the SPDE models (Cox-SPDE and P-SPDE) with the INLA algorithm to optimize computation times [37, 38].

\section{Generalized Additive Model (GAM)}

The GAM model was originally designed to study nonlinear links [39], usually with spline functions. In our particular case, a bivariate spline function of the geographic coordinates of individuals (latitude and longitude) was used to account for spatial heterogeneity of environmental risk $[36,40]$ :

$$
\lambda(t, X)=\lambda_{0}(t) \exp \left(\beta_{1} X_{1}+\beta_{2} X_{2}+\ldots+\beta_{n} X_{n}+f(\text { long, lat })\right)
$$

for each point data, $f$ was a bivariate spline function modeling the spatial effect, long was longitude, and lat was latitude.

\section{SPDE model}

The SPDE model was originally designed to model spatial structures using a Gaussian field [41]. This Bayesian model is generally written as follows:

$$
\begin{aligned}
& Y \mid \beta, X, Z \sim \mathbb{P}(Y \mid \mu, \phi) \\
& Z \sim G F(0, \Sigma)
\end{aligned}
$$

$\mathbb{P}$ is the distribution of $Y$ (dependent variable), $X$ is the vector of covariates (fixed effects), $Z$ is a Gaussian field (GF) with a Matèrn covariance function $\Sigma$ (random spatial effect), and $\mu=E(Y)$ and $\phi$ are the parameters of the distribution of $Y, \mu=E(Y)=h(\beta X+Z)$, where $h$ is the canonical link function.

The Matèrn covariance function is used to control for spatial dependence and for the regularity of the Gaussian field (see Additional file 2). Other covariance functions, 
including exponential ones, are particular cases of the Matèrn covariance function [42]. In our particular case, we applied the SPDE model in two different ways to model outcome distribution: We applied a spatial Cox$\mathrm{PH}$ model (Cox-SPDE) to model survival time, and a Poisson model (P-SPDE) to discretize survival time and to model the number of events within each time interval (Poisson distribution).

\section{Cox-SPDE model}

The Cox-SPDE model is the classic Cox-PH model to which a Gaussian field is added to model the spatial structure $[38,41]$. In our particular case, the dependent variable $(Y)$ was survival time:

$$
\lambda(t, X)=\lambda_{0}(t) \exp \left(\beta_{1} X_{1}+\beta_{2} X_{2}+\ldots+\beta_{n} X_{n}+Z\right)
$$

\section{P-SPDE model}

The P-SPDE model is a piecewise exponential model driven by a counting process [41, 43, 44]. Thus, for a number of events $D$ (where $\delta_{i k}$ is the indicator of the event in individual $i=1,2, \ldots, n$ in the time interval $k=$ $1,2, \ldots, D$, and where $T_{i k}$ is survival time in individual $i$ in the $k^{\text {th }}$ interval), $\delta$ is assumed to follow a Poisson distribution of parameter $\pi$ (average number of events per time interval).

In our particular case, the spatial structure was modeled with a Gaussian field, as earlier described. The dependent variable was the number of events per time interval instead of survival time.

$$
\begin{aligned}
& \delta \sim \text { Poisson }(\pi) \\
& \log (\pi)=\lambda_{0}+\beta_{1} X_{1}+\beta_{2} X_{2}+\ldots+\beta_{n} X_{n}+\log (T)+Z
\end{aligned}
$$

\section{Model performance measures}

The parameter $\beta$ represented the true effect of each covariate estimated by $\hat{\beta}$, with a standard deviation $\overline{S d(\hat{\beta})}$

estimated on $K$ datasets for each scenario and with a 95\% Confidence Interval $C I(\beta)$.

The models were compared using four performance measures [24, 45]: estimation bias $B(\hat{\beta})$, mean square error (MSE), coverage rate (CR), and significance rate (SR), as defined in Additional file 2.

\section{Application}

The four models (Cox-PH, GAM, Cox-SPDE, P-SPDE) were applied, according to the above specifications, to data from a vaccine trial aimed at testing a malaria transmission blocking vaccine candidate: Pfs230 [46]. This randomized controlled trial was conducted in 2015-2016 in the town of Bancoumana, Mali (for more details, see [47]). In our study, the event of interest was a clinical malaria episode. The age, sex, and geographical coordinates (GPS) of participants were also collected. The preventive fraction of the vaccine was calculated based on an estimated prevalence of $77 \%$.

\section{Results}

\section{Data-Generating Model (DGM)}

The DGM was the simulation model, which took into account environmental risk. As expected, biases and MSEs were almost null and nearly always had the desired CR, regardless of the scenario (Additional file 3).

\section{Impact of baseline risk on estimates}

The baseline risk level had roughly no impact on the performance measures of the different models, independently of other parameters (Additional file 4: Figure S2). For example, with a weak breeding site effect (Relative Risk $\left.R R_{b}=1.05\right)$, a low breeding site density $\left(D_{b}=0.25\right)$, a moderate treatment effect (Relative Risk $R R_{t}=0.6$ ), and a low population density $(\tau=0.2)$, performance measures were identical for a baseline risk of 0.06 and a baseline risk of 0.6 for both the Cox-PH model (bias $=-9.3710^{-3}$ and $-8.1410^{-3}$, respectively) and the Cox-SPDE model (bias = $-26.5710^{-3}$ and $-21.3810^{-3}$, respectively).

Similarly, for the age and sex effects, performance measures were little impacted by changes in baseline risk, regardless of the scenario.

The following results were obtained with a baseline risk of 0.37 .

\section{Estimates of the treatment effect}

When the breeding site effect was weak (Relative Risk $R R_{b} \leq 1.2$ ), breeding site density had little impact on the bias and MSE of the treatment effect, which were low for all models, regardless of the scenario.

Thus, with a weak breeding site effect $\left(R R_{b} \leq 1.2\right)$, the bias of the treatment effect ranged from -0.034 to 0.045 for all models except the Cox-SPDE model, regardless of the scenario (G1 and G2 in Figures 3 and Additional file 5: Figure S3).

Under these conditions of weak breeding site effect, the Cox-SPDE model tended to slightly overestimate the treatment effect when population density was low $(\tau=0.2)$, all the more so when the treatment effect was strong (Table 1 ) . For example, with $R R_{t}=0.25, R R_{b}=1.05, D_{b}=0.25$, and $\tau=0.2$, the Cox-SPDE model overestimated the treatment effect with a maximum bias of -0.071 .By contrast, with a high population density $(\tau=0.8$, other parameters remaining unchanged), the bias of the treatment effect was -0.033 . Yet even under conditions of low population density, this overestimation was corrected when the treatment effect was weak $\left(R R_{t} \geq 0.80\right)$. For example, for the CoxSPDE model, with $R R_{b}=1.05, D_{b}=0.25$, and $\tau=0.2$, the 


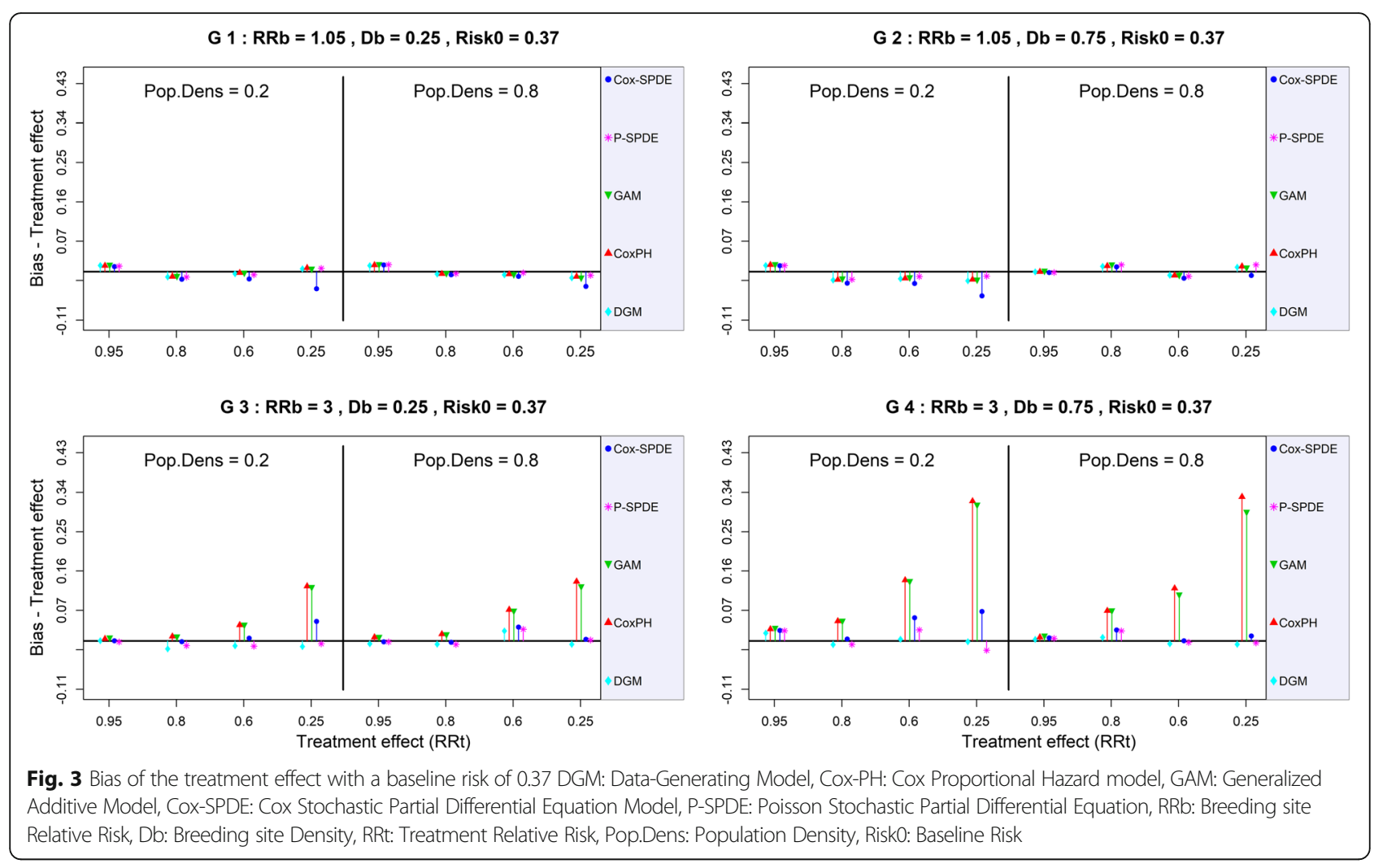

bias of the treatment effect varied between -0.011 and 0.012 for $R R_{t}=0.95$.

The SR (resp. CR) of the treatment effect was similar between all models regardless of the weak breeding site effect scenario. It ranged from $2 \%$ to $24 \%$ (resp. from $86 \%$ to $100 \%$ ) for $R R_{t}=0.95$, and reached $100 \%$ (resp. from $82 \%$ to $100 \%$ ) for $R R_{t}=0.25$. As expected, there was a lack of power when the treatment effect was weak $\left(R R_{t}=0.95\right)(\mathrm{G} 1$ and G2 in Figures 4 and 5).

By contrast, when the breeding site effect was strong $\left(R R_{b} \geq 1.51\right)$, the increase in breeding site density caused an important increase in the bias of the treatment effect for the Cox-PH and GAM models. The bias of the treatment effect was even higher when this effect was strong (G3 and G4 in Figure 3).

For example, for the Cox-PH model, with $R R_{b}=3$, $D_{b}=0.25$, and $\tau=0.2$, the bias of the treatment effect could reach a maximum of 0.139 for the highest level of treatment effect $\left(R R_{t}=0.25\right)$.

With these same parameters, the bias of the treatment effect reached a maximum of 0.131 for the GAM model. As for the two SPDE models, the bias of the treatment effect reached a maximum of 0.054 for the Cox-SPDE model and a maximum of 0.010 for the P-SPDE model.

With a higher breeding site density $\left(D_{b}=0.75\right.$, other parameters remaining unchanged), the bias of the treatment effect reached a maximum of 0.342 for the Cox-PH model for the highest level of treatment effect. The bias of the treatment effect reached a maximum of 0.33 for the GAM model. As for the two SPDE models, the bias of the treatment effect reached a maximum of 0.09 for the Cox-SPDE model and a maximum of 0.014 for the P-SPDE model.

When the breeding site effect was strong $\left(R R_{b} \geq 1.51\right)$, the $C R$ of the treatment effect was negatively impacted by an increase in breeding site density for the Cox-PH and GAM models (G3 and G4 in Figures 4 and 5). This impact was even greater when the treatment effect was strong.

For example, with a strong breeding site effect $R R_{b}=3$ and a high breeding site density $\left(D_{b}=0.75\right)$, the maximum CR of the treatment effect varied between $94 \%$ to $8 \%$ for the Cox-PH model, between $92 \%$ to $14 \%$ for the GAM model, between $98 \%$ to $96 \%$ for the Cox-SPDE model, and between $100 \%$ to $96 \%$ for the P-SPDE model.

The SR of the treatment effect was not impacted by strong breeding site effects. It remained similar between all models, but varied from one scenario to the next depending on the level of treatment effect (with an expected lack of power for the weak treatment effect $\left.R R_{t}=0.95\right)$. Indeed, for the highest level of treatment effect $\left(R R_{t}=0.25\right)$, the SR of the treatment effect varied between $98 \%$ and $100 \%$ for all models. 
Table 1: Model performance measures for estimation of the treatment effect (baseline risk 0.37)

\begin{tabular}{|c|c|c|c|c|c|c|c|c|c|c|c|c|c|c|}
\hline \multirow[t]{5}{*}{$\mathrm{RRb}$} & \multirow[t]{5}{*}{ RRt } & \multirow[t]{5}{*}{ Model } & \multicolumn{12}{|c|}{ Breeding site density } \\
\hline & & & \multicolumn{6}{|l|}{0.25} & \multicolumn{6}{|l|}{0.75} \\
\hline & & & \multicolumn{6}{|c|}{ Population density } & \multicolumn{6}{|c|}{ Population density } \\
\hline & & & \multicolumn{3}{|l|}{0.2} & \multicolumn{3}{|l|}{0.8} & \multicolumn{3}{|l|}{0.2} & \multicolumn{3}{|l|}{0.8} \\
\hline & & & Bias & $C R$ & $S R$ & Bias & $C R$ & $S R$ & Bias & $C R$ & SR & Bias & $C R$ & $S R$ \\
\hline \multirow[t]{16}{*}{1.05} & \multirow[t]{4}{*}{0.95} & $\mathrm{Cox}-\mathrm{PH}$ & 0.014 & 0.96 & 0.06 & 0.015 & 0.88 & 0.08 & 0.016 & 0.92 & 0.06 & $|<0.001|$ & 0.96 & 0.04 \\
\hline & & GAM & 0.014 & 0.98 & 0.06 & 0.015 & 0.86 & 0.08 & 0.015 & 0.92 & 0.06 & $|<0.001|$ & 0.94 & 0.04 \\
\hline & & COX-SPDE & 0.012 & 0.98 & 0.08 & 0.016 & 0.86 & 0.08 & 0.014 & 0.94 & 0.06 & -0.002 & 0.96 & 0.04 \\
\hline & & P-SPDE & 0.013 & 0.98 & 0.08 & 0.016 & 0.86 & 0.1 & 0.014 & 0.94 & 0.06 & -0.002 & 0.96 & 0.04 \\
\hline & \multirow[t]{4}{*}{0.80} & $\mathrm{Cox}-\mathrm{PH}$ & -0.011 & 0.98 & 0.74 & -0.005 & 0.98 & 0.92 & -0.018 & 0.96 & 0.92 & 0.013 & 0.96 & 0.76 \\
\hline & & GAM & -0.012 & 0.98 & 0.74 & -0.006 & 0.98 & 0.9 & -0.018 & 0.96 & 0.92 & 0.014 & 0.96 & 0.74 \\
\hline & & Cox-SPDE & -0.017 & 0.94 & 0.74 & -0.007 & 0.98 & 0.9 & -0.026 & 0.94 & 0.92 & 0.011 & 0.96 & 0.76 \\
\hline & & P-SPDE & -0.012 & 0.96 & 0.76 & -0.004 & 0.98 & 0.9 & -0.018 & 0.96 & 0.9 & 0.016 & 0.96 & 0.76 \\
\hline & \multirow[t]{4}{*}{0.60} & Cox-PH & -0.003 & 0.84 & 1.00 & -0.005 & 0.94 & 1.00 & -0.015 & 0.94 & 1.00 & -0.008 & 0.94 & 1.00 \\
\hline & & GAM & -0.004 & 0.88 & 1.00 & -0.008 & 0.94 & 1.00 & -0.015 & 0.96 & 1.00 & -0.01 & 0.94 & 1.00 \\
\hline & & Cox-SPDE & -0.017 & 0.8 & 1.00 & -0.011 & 0.94 & 1.00 & -0.027 & 0.96 & 1.00 & -0.015 & 0.94 & 1.00 \\
\hline & & P-SPDE & -0.007 & 0.88 & 1.00 & -0.002 & 0.94 & 1.00 & -0.011 & 0.98 & 1.00 & -0.011 & 0.96 & 1.00 \\
\hline & \multirow[t]{4}{*}{0.25} & Cox-PH & 0.009 & 0.98 & 1.00 & -0.011 & 0.98 & 1.00 & -0.018 & 0.96 & 1.00 & 0.012 & 0.9 & 1.00 \\
\hline & & GAM & 0.005 & 0.96 & 1.00 & -0.016 & 0.96 & 1.00 & -0.02 & 0.96 & 1.00 & 0.007 & 0.92 & 1.00 \\
\hline & & COX-SPDE & -0.039 & 0.9 & 1.00 & -0.033 & 0.92 & 1.00 & -0.055 & 0.9 & 1.00 & -0.008 & 0.9 & 1.00 \\
\hline & & P-SPDE & 0.008 & 0.94 & 1.00 & -0.008 & 0.96 & 1.00 & -0.01 & 0.96 & 1.00 & 0.016 & 0.96 & 1.00 \\
\hline \multirow[t]{16}{*}{3} & \multirow[t]{4}{*}{0.95} & Cox-PH & 0.005 & 1.00 & 0.02 & 0.009 & 0.94 & 0.06 & 0.027 & 0.96 & 0.04 & 0.009 & 0.94 & 0.06 \\
\hline & & GAM & 0.005 & 1.00 & 0.02 & 0.007 & 0.94 & 0.08 & 0.027 & 0.96 & 0.04 & 0.01 & 0.98 & 0.08 \\
\hline & & Cox-SPDE & $|<0.001|$ & 1.00 & 0.02 & -0.002 & 0.94 & 0.08 & 0.024 & 0.92 & 0.04 & 0.007 & 1.00 & 0.04 \\
\hline & & P-SPDE & -0.002 & 1.00 & 0.02 & -0.002 & 0.94 & 0.08 & 0.023 & 0.9 & 0.04 & 0.006 & 1.00 & 0.04 \\
\hline & \multirow[t]{4}{*}{0.80} & Cox-PH & 0.01 & 0.96 & 0.78 & 0.016 & 0.92 & 0.8 & 0.045 & 0.92 & 0.6 & 0.069 & 0.84 & 0.5 \\
\hline & & GAM & 0.008 & 0.98 & 0.78 & 0.013 & 0.92 & 0.8 & 0.044 & 0.94 & 0.58 & 0.068 & 0.86 & 0.5 \\
\hline & & Cox-SPDE & -0.002 & 0.98 & 0.78 & -0.003 & 0.96 & 0.82 & 0.004 & 1.00 & 0.64 & 0.025 & 0.98 & 0.48 \\
\hline & & P-SPDE & -0.011 & 0.98 & 0.8 & -0.008 & 0.96 & 0.82 & -0.008 & 1.00 & 0.64 & 0.023 & 1.00 & 0.5 \\
\hline & \multirow[t]{4}{*}{0.60} & Cox-PH & 0.036 & 0.9 & 1.00 & 0.072 & 0.86 & 1.00 & 0.139 & 0.62 & 1.00 & 0.121 & 0.64 & 1.00 \\
\hline & & GAM & 0.035 & 0.92 & 1.00 & 0.067 & 0.86 & 1.00 & 0.135 & 0.6 & 1.00 & 0.104 & 0.78 & 1.00 \\
\hline & & Cox-SPDE & 0.006 & 0.96 & 1.00 & 0.032 & 0.94 & 1.00 & 0.053 & 0.92 & 1.00 & $|<0.001|$ & 0.96 & 1.00 \\
\hline & & P-SPDE & -0.012 & 0.96 & 1.00 & 0.027 & 0.92 & 1.00 & 0.025 & 0.92 & 1.00 & -0.003 & 0.96 & 1.00 \\
\hline & \multirow[t]{4}{*}{0.25} & Cox-PH & 0.125 & 0.78 & 1.00 & 0.136 & 0.58 & 1.00 & 0.32 & 0.02 & 1.00 & 0.33 & 0.06 & 1.00 \\
\hline & & GAM & 0.121 & 0.78 & 1.00 & 0.123 & 0.68 & 1.00 & 0.309 & 0.02 & 1.00 & 0.293 & 0.1 & 1.00 \\
\hline & & Cox-SPDE & 0.045 & 0.94 & 1.00 & 0.004 & 0.98 & 1.00 & 0.067 & 0.94 & 1.00 & 0.011 & 0.94 & 1.00 \\
\hline & & P-SPDE & -0.006 & 1.00 & 1.00 & 0.002 & 1.00 & 1.00 & -0.021 & 0.98 & 1.00 & -0.004 & 0.92 & 1.00 \\
\hline
\end{tabular}

CR Coverage Rate, SR Significance Rate, Cox-PH Cox Proportional Hazard model, GAM Generalized Additive Model, Cox-SPDE Cox-Stochastic Partial Differential Equation Model, $P$-SPDE Poisson-Stochastic Partial Differential Equation Model, RRt Treatment Relative Risk, RRb Breeding site Relative Risk. See Additional file 6 for MSE Mean Square Error

\section{Application}

In the context of the Pfs230 vaccine trial, none of the models yielded a significant vaccine effect on clinical episodes. The Cox-SPDE model estimated a slightly higher preventive fraction $(29.26 \%$ [-10.93\%; 51.21\%]), followed closely by the P-SPDE model (26.95\% [-15.25\%; 49.90\%]) . By contrast, the non-spatial Cox-PH model estimated a slightly lower preventive fraction (24.64\% [-16.56\%; $47.66 \%])$, as did the GAM model (22.33\% [-22.25\%; $46.82 \%])$.

\section{Estimates of the age effect}

When the breeding site effect was weak $\left(R R_{b}=1.05\right.$ or 1.2 ), breeding site density had little impact on the bias 


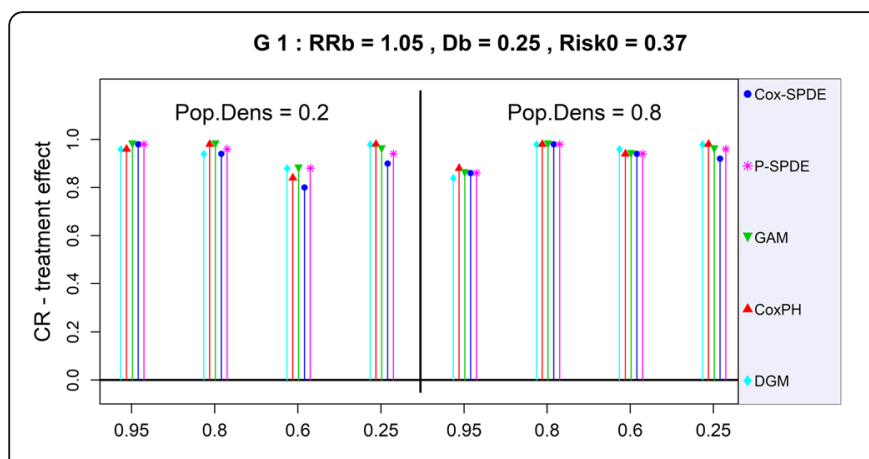

$\mathrm{G} 3: \mathrm{RRb}=3, \mathrm{Db}=0.25$, Risk $0=0.37$
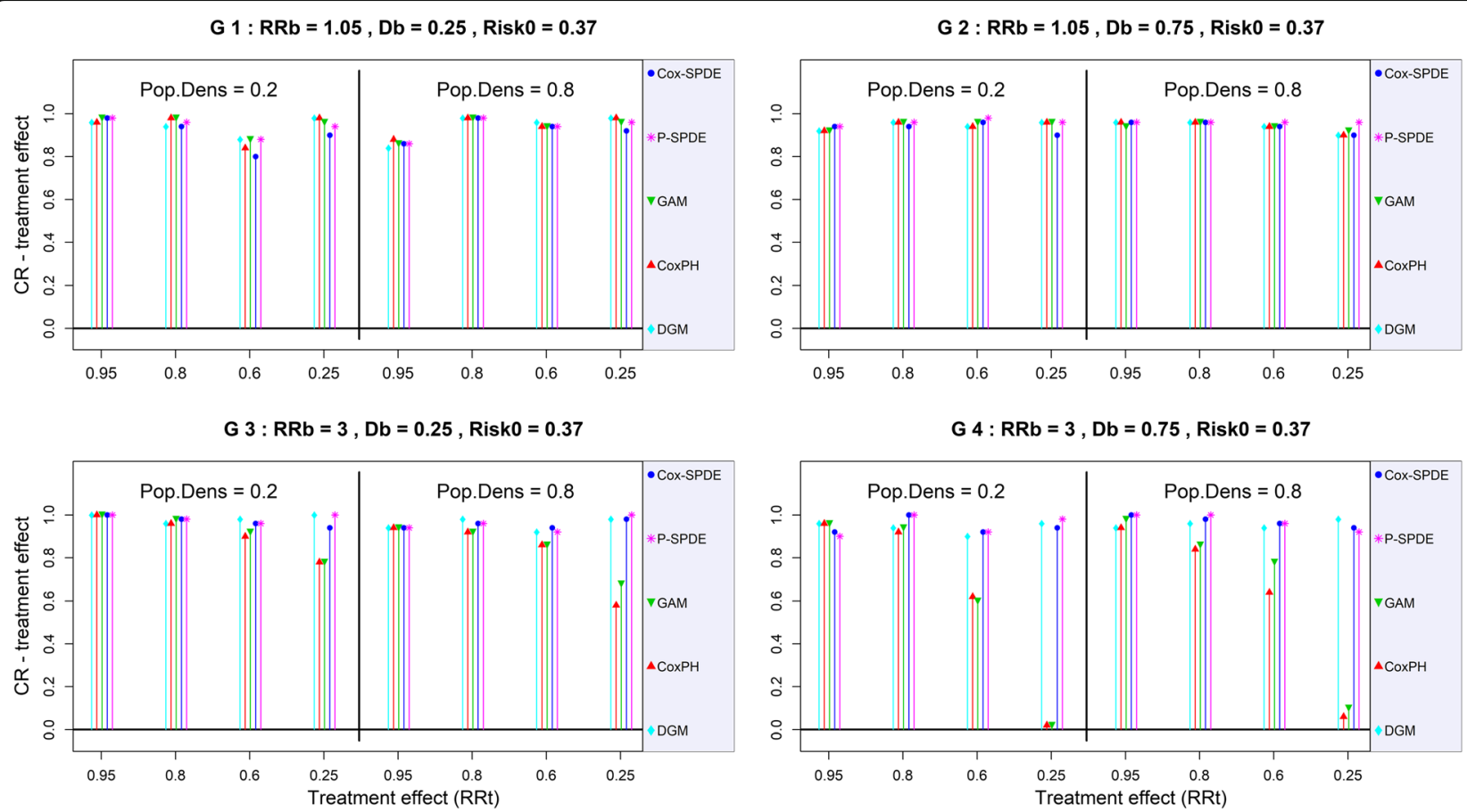

Fig. 4 CR of the treatment effect with a baseline risk of 0.37. DGM: Data-Generating Model, Cox-PH: Cox Proportional Hazard model, GAM: Generalized Additive Model, Cox-SPDE: Cox Stochastic Partial Differential Equation Model, P-SPDE: Poisson Stochastic Partial Differential Equation, RRb: Breeding site Relative Risk, Db: Breeding site Density, RRt: Treatment Relative Risk, Pop.Dens: Population Density, Risk0: Baseline Risk.

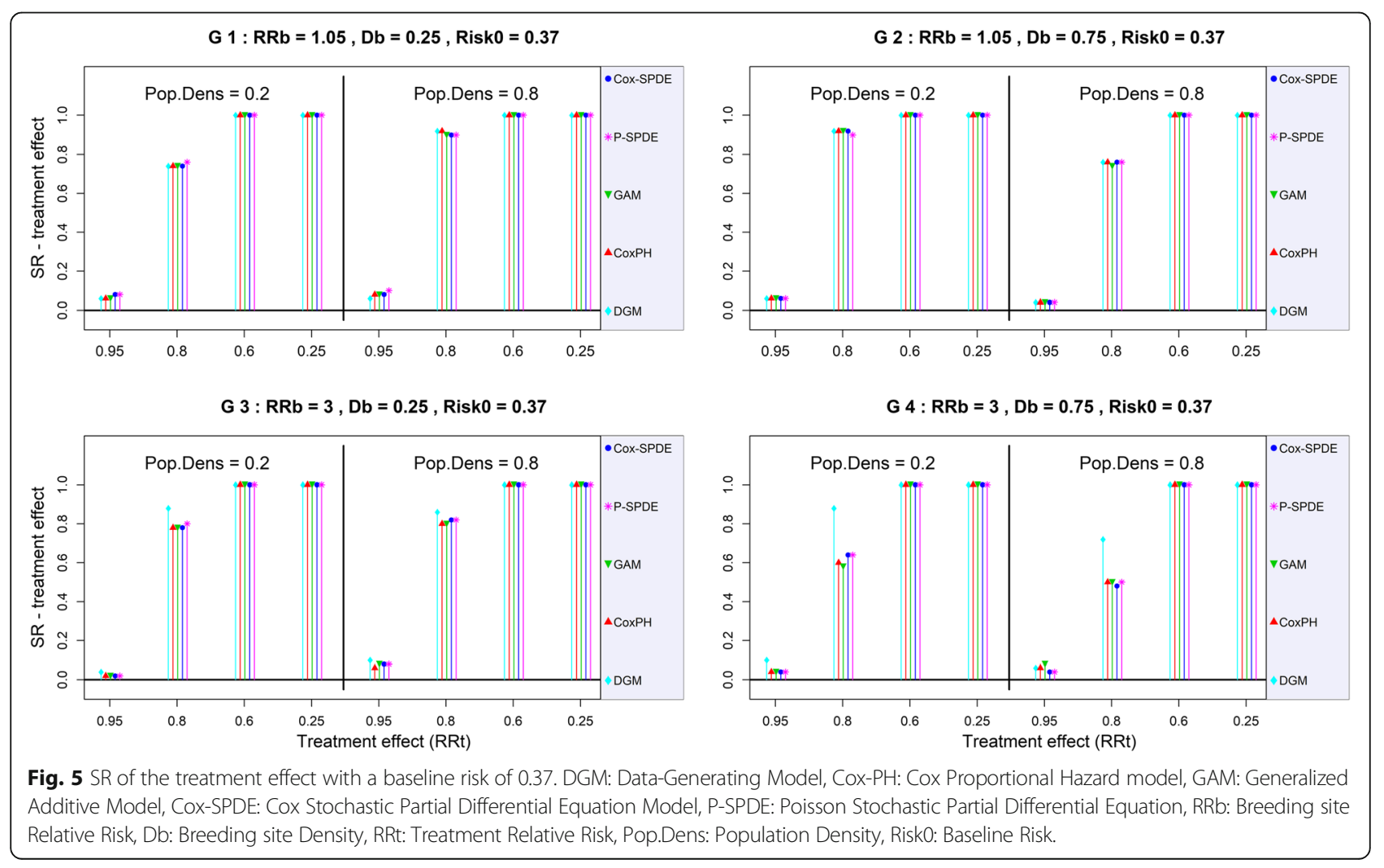


Table 2: Model performance measures for estimation of the age effect (baseline risk 0.37)

\begin{tabular}{|c|c|c|c|c|c|c|c|c|c|c|c|c|c|c|}
\hline \multirow[t]{5}{*}{$\mathrm{RRb}$} & \multirow[t]{5}{*}{ RRt } & \multirow[t]{5}{*}{ Model } & \multicolumn{12}{|c|}{ Breeding site density } \\
\hline & & & \multicolumn{6}{|c|}{0.25} & \multicolumn{6}{|l|}{0.75} \\
\hline & & & \multicolumn{6}{|c|}{ Population density } & \multicolumn{6}{|c|}{ Population density } \\
\hline & & & \multicolumn{3}{|l|}{0.2} & \multicolumn{3}{|l|}{0.8} & \multicolumn{3}{|l|}{0.2} & \multicolumn{3}{|l|}{0.8} \\
\hline & & & Bias & $C R$ & $S R$ & Bias & $C R$ & SR & Bias & $C R$ & $S R$ & Bias & $C R$ & $S R$ \\
\hline \multirow[t]{16}{*}{1.05} & \multirow[t]{4}{*}{0.95} & $\mathrm{COx}-\mathrm{PH}$ & 0.002 & 0.96 & 1.00 & $|<0.001|$ & 0.9 & 1.00 & -0.002 & 0.98 & 1.00 & -0.001 & 0.98 & 1.00 \\
\hline & & GAM & 0.001 & 0.94 & 1.00 & -0.001 & 0.9 & 1.00 & -0.002 & 0.96 & 1.00 & -0.002 & 0.96 & 1.00 \\
\hline & & Cox-SPDE & -0.004 & 0.76 & 1.00 & -0.003 & 0.86 & 1.00 & -0.006 & 0.72 & 1.00 & -0.003 & 0.88 & 1.00 \\
\hline & & P-SPDE & $|<0.001|$ & 0.94 & 1.00 & -0.001 & 0.96 & 1.00 & -0.001 & 0.96 & 1.00 & -0.001 & 0.92 & 1.00 \\
\hline & \multirow[t]{4}{*}{0.80} & $\mathrm{Cox}-\mathrm{PH}$ & $|<0.001|$ & 0.96 & 1.00 & -0.001 & 0.96 & 1.00 & $|<0.001|$ & 0.96 & 1.00 & -0.002 & 0.84 & 1.00 \\
\hline & & GAM & $|<0.001|$ & 0.96 & 1.00 & -0.002 & 0.96 & 1.00 & -0.001 & 0.98 & 1.00 & -0.003 & 0.88 & 1.00 \\
\hline & & Cox-SPDE & -0.004 & 0.76 & 1.00 & -0.003 & 0.86 & 1.00 & -0.006 & 0.68 & 1.00 & -0.005 & 0.72 & 1.00 \\
\hline & & P-SPDE & -0.001 & 0.96 & 1.00 & -0.001 & 0.92 & 1.00 & $|<0.001|$ & 0.94 & 1.00 & -0.002 & 0.92 & 1.00 \\
\hline & \multirow[t]{4}{*}{0.60} & $\mathrm{COx}-\mathrm{PH}$ & 0.001 & 0.9 & 1.00 & -0.001 & 0.96 & 1.00 & -0.002 & 0.96 & 1.00 & 0.001 & 0.96 & 1.00 \\
\hline & & GAM & $|<0.001|$ & 0.92 & 1.00 & -0.002 & 0.96 & 1.00 & -0.002 & 0.94 & 1.00 & $|<0.001|$ & 0.96 & 1.00 \\
\hline & & Cox-SPDE & -0.003 & 0.84 & 1.00 & -0.003 & 0.86 & 1.00 & -0.007 & 0.66 & 1.00 & -0.002 & 0.88 & 1.00 \\
\hline & & P-SPDE & $|<0.001|$ & 0.94 & 1.00 & -0.001 & 0.94 & 1.00 & -0.002 & 0.98 & 1.00 & $|<0.001|$ & 0.94 & 1.00 \\
\hline & \multirow[t]{4}{*}{0.25} & $\mathrm{Cox}-\mathrm{PH}$ & $|<0.001|$ & 0.98 & 1.00 & -0.001 & 0.98 & 1.00 & -0.001 & 0.94 & 1.00 & $|<0.001|$ & 0.96 & 1.00 \\
\hline & & GAM & $|<0.001|$ & 0.98 & 1.00 & -0.003 & 0.9 & 1.00 & -0.002 & 0.92 & 1.00 & $|<0.001|$ & 0.94 & 1.00 \\
\hline & & COX-SPDE & -0.006 & 0.66 & 1.00 & -0.004 & 0.8 & 1.00 & -0.006 & 0.76 & 1.00 & -0.003 & 0.8 & 1.00 \\
\hline & & P-SPDE & $|<0.001|$ & 0.96 & 1.00 & -0.001 & 0.96 & 1.00 & $|<0.001|$ & 0.94 & 1.00 & $|<0.001|$ & 1.00 & 1.00 \\
\hline \multirow[t]{16}{*}{3} & \multirow[t]{4}{*}{0.95} & Cox-PH & 0.018 & 0.16 & 1.00 & 0.016 & 0.32 & 1.00 & 0.041 & 0.00 & 1.00 & 0.042 & 0.00 & 1.00 \\
\hline & & GAM & 0.017 & 0.2 & 1.00 & 0.014 & 0.5 & 1.00 & 0.04 & 0.00 & 1.00 & 0.037 & 0.00 & 1.00 \\
\hline & & COX-SPDE & 0.007 & 0.74 & 1.00 & $|<0.001|$ & 0.98 & 1.00 & 0.01 & 0.84 & 1.00 & 0.003 & 0.86 & 1.00 \\
\hline & & P-SPDE & -0.001 & 0.92 & 1.00 & -0.001 & 0.96 & 1.00 & $|<0.001|$ & 0.94 & 1.00 & $|<0.001|$ & 0.96 & 1.00 \\
\hline & \multirow[t]{4}{*}{0.80} & $\mathrm{COX}-\mathrm{PH}$ & 0.018 & 0.18 & 1.00 & 0.019 & 0.18 & 1.00 & 0.042 & 0.00 & 1.00 & 0.041 & 0.00 & 1.00 \\
\hline & & GAM & 0.017 & 0.26 & 1.00 & 0.018 & 0.32 & 1.00 & 0.04 & 0.00 & 1.00 & 0.036 & 0.00 & 1.00 \\
\hline & & Cox-SPDE & 0.007 & 0.86 & 1.00 & 0.005 & 0.9 & 1.00 & 0.011 & 0.8 & 1.00 & 0.002 & 0.98 & 1.00 \\
\hline & & P-SPDE & 0.001 & 0.92 & 1.00 & 0.001 & 0.96 & 1.00 & 0.002 & 0.9 & 1.00 & $|<0.001|$ & 0.96 & 1.00 \\
\hline & \multirow[t]{4}{*}{0.60} & $\mathrm{COx}-\mathrm{PH}$ & 0.017 & 0.26 & 1.00 & 0.016 & 0.3 & 1.00 & 0.042 & 0.00 & 1.00 & 0.04 & 0.00 & 1.00 \\
\hline & & GAM & 0.016 & 0.28 & 1.00 & 0.015 & 0.52 & 1.00 & 0.041 & 0.00 & 1.00 & 0.037 & 0.00 & 1.00 \\
\hline & & Cox-SPDE & 0.007 & 0.8 & 1.00 & 0.001 & 0.94 & 1.00 & 0.012 & 0.76 & 1.00 & 0.001 & 0.96 & 1.00 \\
\hline & & P-SPDE & 0.001 & 0.98 & 1.00 & -0.001 & 0.98 & 1.00 & 0.002 & 0.94 & 1.00 & -0.001 & 0.96 & 1.00 \\
\hline & \multirow[t]{4}{*}{0.25} & $\mathrm{Cox}-\mathrm{PH}$ & 0.018 & 0.2 & 1.00 & 0.016 & 0.28 & 1.00 & 0.042 & 0.00 & 1.00 & 0.043 & 0.00 & 1.00 \\
\hline & & GAM & 0.017 & 0.2 & 1.00 & 0.014 & 0.44 & 1.00 & 0.041 & 0.00 & 1.00 & 0.038 & 0.00 & 1.00 \\
\hline & & Cox-SPDE & 0.007 & 0.8 & 1.00 & -0.001 & 0.94 & 1.00 & 0.011 & 0.78 & 1.00 & 0.003 & 0.92 & 1.00 \\
\hline & & P-SPDE & 0.001 & 0.96 & 1.00 & -0.001 & 0.92 & 1.00 & $|<0.001|$ & 0.98 & 1.00 & 0.001 & 0.94 & 1.00 \\
\hline
\end{tabular}

CR Coverage Rate, SR Significance Rate, Cox-PH Cox Proportional Hazard model, GAM Generalized Additive Model, Cox-SPDE Cox-Stochastic Partial Differential Equation Model, $P$-SPDE Poisson-Stochastic Partial Differential Equation Model, RRt Treatment Relative Risk, RRb Breeding site Relative Risk. See Additional file 6 for MSE Mean Square Error

and MSE of the age effect, regardless of the model. For example, regardless of the weak breeding site effect scenario, the bias of the age effect was around -0.0035 for all models except the Cox-SPDE model (G1 and G2 in Additional file 7: Figure S4 and Additional file 8: Figure S5). As was observed for the treatment effect, the latter model tended to slightly overestimate the age effect, all the more so when population density in the clusters was low. Thus, for a population density $\tau=0.2$, the bias was around -0.007 (Table 2).

The SR of the age effect was identical for all models regardless of the scenario. However, the CR of 
the age effect was slightly lower for the Cox-SPDE model when population density was low. Indeed, with a weak breeding site effect $\left(R R_{b}=1.05\right)$, a low breeding site density $\left(D_{b}=0.25\right)$, and a population density $\tau=0.2$, the CR of the age effect ranged from $86 \%$ to $100 \%$ for the non-spatial Cox-PH model and from $62 \%$ to $92 \%$ for the Cox-SPDE model.

Conversely, when the breeding site effect was strong $\left(R R_{b}=1.51\right.$ and 3$)$, the bias of the age effect increased markedly with breeding site density $D_{b}$ for the Cox-PH and GAM models (G3 and G4 in Additional file 7: Figure S4 and Additional file 8: Figure S5). For example, for the Cox-PH model, with a strong breeding site effect $\left(R R_{b}=3\right)$ and a low population density $(\tau=0.2)$, the bias of the age effect was around 0.017 for a low breeding site density $\left(D_{b}=0.25\right)$ and around 0.041 for a high breeding site density $\left(D_{b}=0.75\right)$.

The bias of the age effect was very similar between the GAM model and the Cox-PH model, regardless of the scenario. For the Cox-SPDE model, the bias of the age effect was slightly impacted by population density: it was lower when this density was high. With a strong breeding site effect $\left(R R_{b}=3\right)$ and a high breeding site density $\left(D_{b}=0.75\right)$, the bias of the age effect was around 0.01 for a low population density $(\tau=0.2)$ and around 0.001 for a high population density $(\tau=0.8)$. For the P-SPDE model, the bias of the age effect was low regardless of the scenario $(\sim 0)$. It should be noted that the bias of the age effect was similar between the P-SPDE model and the Cox-SPDE model, all the more so when population density was high.

The CR of the age effect was low for the Cox-PH and GAM models (G3 and G4 in Additional file 9: Figure S6). For example, for the Cox-PH model (resp. GAM model), with a strong breeding site effect $\left(R R_{b}=3\right)$, the $\mathrm{CR}$ of the age effect was $0 \%$ (resp. $<2 \%$ ) when breeding site density was high $\left(D_{b}=0.75\right)$. Under these same conditions, for the Cox-SPDE and P-SPDE models, the CR of the age effect remained high at $82 \%$ to $100 \%$, respectively. However, the SR of the age effect was $100 \%$ for all models, regardless of the scenario (Additional file 10: Figure S7).

It is important to note that for these simulated datasets, the level of treatment effect had no impact on the estimates of the age effect, regardless of the scenario.

\section{Estimates of the sex effect}

Regardless of the scenario, the bias, MSE, CR, and SR of the sex effect were roughly identical for all models (Table 3), with very little variation from one scenario to the next (Additional file 11: Figure S8, Additional file 12: Figure S9, Additional file 13: Figure S10, Additional file 14: Figure S11).

\section{Discussion}

The aim of this study was to highlight the impact of the spatial heterogeneity of non-measured environmental risk on the results of prevention trials by using simulated data in different scenarios. We have shown that despite randomization, spatial heterogeneity leads to underestimating the treatment effect, with a CR that is almost null in some situations. In our study, the conditions leading to the most biased results were strong environmental effect (breeding site effect in our application context) in conjunction with high environmental density (breeding site density in our application context).

This is unsurprising, as environmental risk is known to vary according to the location of individuals and to environmental factors [48]. Bias correction was achieved with models that accounted for the location of individuals as a proxy for variation in environmental risk, in particular with the P-SPDE model.

The limits of the breeding site effect $R R_{b}$ were set between 1.05 and 3 and those of breeding site density $D_{b}$ (which expressed the probability of being exposed to at least one breeding site) were set between 0.25 and 0.75 . Bias increased linearly, and was important starting from $R R_{b}=1.5$, even when breeding site density was low $\left(D_{b}=\right.$ $0.25)$. However, scenarios with a high breeding site density $\left(D_{b}=0.75\right)$ and a weak breeding site effect $\left(R R_{b}\right.$ $=1.05,1.20)$ did not yield a clear bias.

Despite randomization, the treatment effect also impacted the estimates. Indeed, bias increased linearly with the treatment effect, except when the breeding site effect was weak $\left(R R_{b} \leq 1.2\right)$. With a strong treatment effect (Relative Risk $R R_{t} \leq 0.6$ ), a strong breeding site effect $\left(R R_{b} \geq 1.5\right)$, and a high breeding site density $\left(D_{b}=0.75\right)$, underestimation was maximal and CR was almost null.

Neither baseline risk nor population density had a strong impact on the quality of the estimates. The range of values selected for the baseline risk was within the limits observed in our application context (malaria). However, the absence of an effect of baseline risk was expected because baseline risk applied uniformly to the entire study zone, without influencing the estimates.

In our application context, environmental risk depended little on population density; it depended mainly on breeding site density, as breeding sites are the source of malaria vectors. Our results would likely have been different if the studied pathology had been highly dependent on population density (e.g., cholera).

As expected, no significant effect was observed in the context of the vaccine trial. The absence of a significant vaccine effect on malaria episodes may explain why the difference in estimates between the different models was small. However, these estimates were consistent with our simulations results, as the effects estimated with the Cox- 
Table 3: Model performance measures for estimation of the sex effect (baseline risk 0.37)

\begin{tabular}{|c|c|c|c|c|c|c|c|c|c|c|c|c|c|c|}
\hline \multirow[t]{5}{*}{$\mathrm{RRb}$} & \multirow[t]{5}{*}{ RRt } & \multirow[t]{5}{*}{ Model } & \multicolumn{12}{|c|}{ Breeding site density } \\
\hline & & & \multicolumn{6}{|c|}{0.25} & \multicolumn{6}{|l|}{0.75} \\
\hline & & & \multicolumn{6}{|c|}{ Population density } & \multicolumn{6}{|c|}{ Population density } \\
\hline & & & \multicolumn{3}{|l|}{0.2} & \multicolumn{3}{|l|}{0.8} & \multicolumn{3}{|l|}{0.2} & \multicolumn{3}{|l|}{0.8} \\
\hline & & & Bias & $C R$ & SR & Bias & $C R$ & $S R$ & Bias & $C R$ & $S R$ & Bias & $C R$ & $S R$ \\
\hline \multirow[t]{16}{*}{1.05} & \multirow[t]{4}{*}{0.95} & $\mathrm{Cox}-\mathrm{PH}$ & -0.009 & 1.00 & 0.00 & -0.022 & 0.88 & 0.12 & -0.013 & 0.96 & 0.04 & 0.004 & 0.92 & 0.08 \\
\hline & & GAM & -0.009 & 1.00 & 0.00 & -0.021 & 0.88 & 0.12 & -0.013 & 0.96 & 0.04 & 0.005 & 0.92 & 0.08 \\
\hline & & Cox-SPDE & -0.009 & 1.00 & 0.00 & -0.024 & 0.88 & 0.12 & -0.015 & 0.96 & 0.04 & 0.006 & 0.96 & 0.04 \\
\hline & & P-SPDE & -0.008 & 1.00 & 0.00 & -0.024 & 0.88 & 0.12 & -0.014 & 0.96 & 0.04 & 0.005 & 0.96 & 0.04 \\
\hline & \multirow[t]{4}{*}{0.80} & $\mathrm{Cox}-\mathrm{PH}$ & -0.01 & 0.96 & 0.04 & $|<0.001|$ & 0.96 & 0.04 & 0.012 & 0.96 & 0.04 & 0.011 & 0.92 & 0.08 \\
\hline & & GAM & -0.01 & 0.98 & 0.02 & $|<0.001|$ & 0.98 & 0.02 & 0.013 & 0.96 & 0.04 & 0.012 & 0.92 & 0.08 \\
\hline & & Cox-SPDE & -0.01 & 0.96 & 0.04 & 0.001 & 0.98 & 0.02 & 0.014 & 0.96 & 0.04 & 0.013 & 0.94 & 0.06 \\
\hline & & P-SPDE & -0.011 & 0.96 & 0.04 & $|<0.001|$ & 0.98 & 0.02 & 0.013 & 0.96 & 0.04 & 0.012 & 0.94 & 0.06 \\
\hline & \multirow[t]{4}{*}{0.60} & $\mathrm{Cox}-\mathrm{PH}$ & -0.024 & 0.98 & 0.02 & 0.004 & 0.98 & 0.02 & 0.002 & 0.92 & 0.08 & $|<0.001|$ & 0.94 & 0.06 \\
\hline & & GAM & -0.024 & 0.98 & 0.02 & 0.003 & 0.98 & 0.02 & 0.003 & 0.92 & 0.08 & 0.002 & 0.94 & 0.06 \\
\hline & & Cox-SPDE & -0.023 & 0.96 & 0.04 & 0.004 & 0.98 & 0.02 & 0.001 & 0.96 & 0.04 & 0.003 & 0.94 & 0.06 \\
\hline & & P-SPDE & -0.023 & 0.96 & 0.04 & 0.005 & 0.98 & 0.02 & 0.001 & 0.98 & 0.02 & 0.003 & 0.94 & 0.06 \\
\hline & \multirow[t]{4}{*}{0.25} & Cox-PH & -0.004 & 0.96 & 0.04 & 0.007 & 0.98 & 0.02 & -0.006 & 0.94 & 0.06 & -0.001 & 0.96 & 0.04 \\
\hline & & GAM & -0.003 & 0.96 & 0.04 & 0.006 & 0.98 & 0.02 & -0.007 & 0.94 & 0.06 & -0.001 & 0.96 & 0.04 \\
\hline & & Cox-SPDE & -0.005 & 0.96 & 0.04 & 0.008 & 0.98 & 0.02 & -0.008 & 0.94 & 0.06 & $|<0.001|$ & 0.94 & 0.06 \\
\hline & & P-SPDE & -0.005 & 0.96 & 0.04 & 0.008 & 0.98 & 0.02 & -0.007 & 0.94 & 0.06 & $|<0.001|$ & 0.96 & 0.04 \\
\hline \multirow[t]{16}{*}{3} & \multirow[t]{4}{*}{0.95} & Cox-PH & 0.002 & 0.98 & 0.02 & -0.019 & 0.92 & 0.08 & -0.026 & 0.92 & 0.08 & 0.003 & 0.96 & 0.04 \\
\hline & & GAM & 0.002 & 0.98 & 0.02 & -0.019 & 0.9 & 0.1 & -0.028 & 0.92 & 0.08 & 0.004 & 0.96 & 0.04 \\
\hline & & Cox-SPDE & 0.002 & 0.96 & 0.04 & -0.022 & 0.9 & 0.1 & -0.039 & 0.96 & 0.04 & 0.013 & 0.94 & 0.06 \\
\hline & & P-SPDE & 0.003 & 0.96 & 0.04 & -0.022 & 0.9 & 0.1 & -0.042 & 0.94 & 0.06 & 0.014 & 0.94 & 0.06 \\
\hline & \multirow[t]{4}{*}{0.80} & $\mathrm{Cox}-\mathrm{PH}$ & 0.004 & 0.94 & 0.06 & -0.008 & 0.94 & 0.06 & -0.006 & 0.96 & 0.04 & -0.004 & 0.96 & 0.04 \\
\hline & & GAM & 0.005 & 0.94 & 0.06 & -0.008 & 0.94 & 0.06 & -0.009 & 0.94 & 0.06 & -0.001 & 0.94 & 0.06 \\
\hline & & Cox-SPDE & 0.005 & 0.92 & 0.08 & -0.009 & 0.98 & 0.02 & -0.01 & 0.96 & 0.04 & 0.002 & 0.96 & 0.04 \\
\hline & & P-SPDE & 0.006 & 0.92 & 0.08 & -0.009 & 0.96 & 0.04 & -0.01 & 0.96 & 0.04 & 0.003 & 0.96 & 0.04 \\
\hline & \multirow[t]{4}{*}{0.60} & Cox-PH & -0.016 & 0.98 & 0.02 & 0.002 & 0.98 & 0.02 & -0.01 & 0.96 & 0.04 & -0.007 & 0.9 & 0.1 \\
\hline & & GAM & -0.018 & 0.98 & 0.02 & 0.003 & 0.96 & 0.04 & -0.01 & 0.98 & 0.02 & -0.004 & 0.92 & 0.08 \\
\hline & & Cox-SPDE & -0.018 & 0.98 & 0.02 & 0.001 & 0.94 & 0.06 & -0.014 & 0.96 & 0.04 & -0.001 & 0.96 & 0.04 \\
\hline & & P-SPDE & -0.018 & 0.98 & 0.02 & 0.001 & 0.92 & 0.08 & -0.013 & 0.92 & 0.08 & -0.001 & 0.96 & 0.04 \\
\hline & \multirow[t]{4}{*}{0.25} & Cox-PH & -0.02 & 0.9 & 0.1 & -0.003 & 0.98 & 0.02 & -0.001 & 0.92 & 0.08 & -0.006 & 0.92 & 0.08 \\
\hline & & GAM & -0.018 & 0.92 & 0.08 & -0.001 & 0.98 & 0.02 & $|<0.001|$ & 0.9 & 0.1 & 0.002 & 0.92 & 0.08 \\
\hline & & Cox-SPDE & -0.015 & 0.9 & 0.1 & -0.003 & 0.96 & 0.04 & 0.007 & 0.96 & 0.04 & -0.002 & 0.92 & 0.08 \\
\hline & & P-SPDE & -0.015 & 0.9 & 0.1 & -0.004 & 0.98 & 0.02 & 0.009 & 0.96 & 0.04 & -0.002 & 0.9 & 0.1 \\
\hline
\end{tabular}

CR Coverage Rate, SR Significance Rate, Cox-PH Cox Proportional Hazard model, GAM Generalized Additive Model, Cox-SPDE Cox-Stochastic Partial Differential Equation Model, P-SPDE Poisson-Stochastic Partial Differential Equation Model, RRt Treatment Relative Risk, RRb Breeding site Relative Risk. See Additional file 6 for MSE Mean Square Error

$\mathrm{PH}$ and GAM models were weaker than those estimated with the Cox-SPDE and P-SPDE models.

In the literature, spatial heterogeneity is usually accounted for with classic mixed models. While these models were not explicitly developed to account for spatial heterogeneity, they can partly capture spatial heterogeneity by aggregating individuals with the same risk profile. One such model is the Structured Additive Regression (STAR) model [49-52], which takes into account both the contiguity structure and the spatial correlation between areas. In this model, the study zone is split into several predefined subdivisions to represent spatial heterogeneity of environmental risk, and environmental risk is then assumed to be homogeneous within each subdivision [7, 53]. However, as our study 
indicates, two close individuals can have different risks depending on the proximity of the risk source, which means that the assumption of homogeneity is not always tenable. This is especially the case when administrative subdivisions are used, or when the studied disease is strongly associated with the environment-for instance malaria, for which finescale heterogeneity of environmental risk has been described [9]. When the study zone is split even more finely, the excessive number of subdivisions to be included in the mixed model leads to an inflation in the number of parameters to be estimated, and the estimate of variation within each overly small subdivision becomes unstable. In other words, this approach involves a choice between the homogeneity hypothesis and the number of parameters, and the yielded results depend on the choice of shape and size of the subdivisions. In the context of vector-borne diseases, the construction of homogeneous risk areas would require observing every single breeding site, which is unrealistic [10]. The aim of our study, then, was not to account for a particular measured spatial structure (to which a STAR model could be applied), but to highlight the impact of spatial structure on study results and to propose different approaches for modeling spatial heterogeneity when spatial risk factors are not precisely measured. Although few studies have accounted for spatial heterogeneity of environmental risk, many have examined temporal variation in risk.

One method used to study this variation is the Cox-PH model, in which the duration of the study is stratified into time intervals so as to obtain periods of homogeneous risk [54, 55]. However, more continuous approaches (especially ones using spline functions) have also been used [56]. An increasing number of studies have used GAM models to account for spatial heterogeneity of risk [39, 57]. In this approach, spatial heterogeneity is modeled with a bivariate spline function of the geographic coordinates of individuals [36]. However, in our study, little difference in performance was found between the GAM model and the Cox-PH model. This may be explained by the fact that the GAM model estimates local risk by aggregating individual data $[40,56]$, when in fact survival approaches (such as the one we used) require that these data be kept separate. Moreover, even when the true spatial sources of environmental risk (breeding sites) are not measured, we know that their location differs from that of individuals. This inaccuracy, along with the mode of estimation of bivariate spline functions, may explain the poor results yielded in our study by the GAM model $[38,41]$. Our best results were obtained with the Cox-SPDE and P-SPDE models, which modeled the spatial effect using a Gaussian field (random spatial effect) with a Matèrn covariance function. In our simulation study, the P-SPDE model remained stable and followed closely the DGM model, regardless of the scenario. However, the Cox-SPDE model was a little sensitive to population density, slightly overestimating parameters when population density was low. Lastly, as the Matèrn function decreased with distance, the spatial effect on survival time became very small. The difference in results between the two SPDE models for low population densities may therefore be explained by the fact that the P-SPDE model was used to model the number of events as opposed to survival time. It should be noted, however, that both models showed better results than the classic Cox-PH model.

\section{Conclusion}

Our study shows that bias due to spatial heterogeneity of environmental risk is not adequately eliminated with randomization. The underestimation of the treatment effect (with almost null CRs in some situations) highlighted in our study may explain why certain treatments or strategies against malaria end up being rejected. Spatial location modeling can reduce bias due to spatial heterogeneity of environmental risk, the latter being sometimes difficult to measure. For this purpose, SPDE models that model spatial heterogeneity with a Gaussian field seem to be the most appropriate.

\section{Additional files}

\begin{abstract}
Additional file 1: Figure S1. Bias of the treatment effect with a baseline risk of 0.37 for 500 simulations. DGM: Data-Generating Model, Cox-PH: Cox Proportional Hazard model, GAM: Generalized Additive Model, Cox-SPDE: Cox-Stochastic Partial Differential Equation Model, PSPDE: Poisson-Stochastic Partial Differential Equation, RRb: Breeding site Relative Risk, Db: Breeding site Density, RRt: Treatment Relative Risk, Pop.Dens: Population Density, Risk0: Baseline Risk. (TIF 782 kb)
\end{abstract}

Additional file 2: Description of mathematical formulas and terms used in this manuscript. (DOCX $17 \mathrm{~kb}$ )

Additional file 3: Data-Generating Model (DGM) performance index for estimating the different factor effects (baseline risk 0.37). (DOCX $23 \mathrm{~kb}$ )

Additional file 4: Figure S2. Bias of the treatment effect with a population density of 0.5. DGM: Data-Generating Model, Cox-PH: Cox Proportional Hazard model, GAM: Generalized Additive Model, Cox-SPDE: Cox-Stochastic Partial Differential Equation Model, P-SPDE: Poisson-Stochastic Partial Differential Equation, RRb: Breeding site Relative Risk, Db: Breeding site Density, RRt: Treatment Relative Risk, Pop.Dens: Population Density, Risk0: Baseline Risk. (TIF 773 kb)

Additional file 5: Figure S3. MSE of the treatment effect with a baseline risk of 0.37. DGM: Data-Generating Model, Cox-PH: Cox Proportional Hazard model, GAM: Generalized Additive Model, Cox-SPDE: CoxStochastic Partial Differential Equation Model, P-SPDE: Poisson-Stochastic Partial Differential Equation, RRb: Breeding site Relative Risk, Db: Breeding site Density, RRt: Treatment Relative Risk, Pop.Dens: Population Density, Risk0: Baseline Risk. (TIF 777 kb)

Additional file 6: MSE of the treatment, age, and sex effect for all models (baseline risk 0.37). (DOCX $32 \mathrm{~kb}$ )

Additional file 7: Figure S4. Bias of the age effect with a baseline risk of 0.37. DGM: Data-Generating Model, Cox-PH: Cox Proportional Hazard model, GAM: Generalized Additive Model, Cox-SPDE: Cox-Stochastic Partial Differential Equation Model, P-SPDE: Poisson-Stochastic Partial Differential Equation, RRb: Breeding site Relative Risk, Db: Breeding site Density, RRt: Treatment Relative Risk, Pop.Dens: Population Density, Risk0: Baseline Risk. (TIF 794 kb)

Additional file 8: Figure S5. MSE of the age effect with a baseline risk of 0.37.DGM: Data-Generating Model, Cox-PH: Cox Proportional Hazard model, GAM: Generalized Additive Model, Cox-SPDE: Cox-Stochastic 
Partial Differential Equation Model, P-SPDE: Poisson-Stochastic Partial Differential Equation, RRb: Breeding site Relative Risk, Db: Breeding site Density, RRt: Treatment Relative Risk, Pop.Dens: Population Density, Risk0: Baseline Risk. (TIF $753 \mathrm{~kb}$ )

Additional file 9: Figure S6. CR of the age effect with a baseline risk of 0.37. DGM: Data-Generating Model, Cox-PH: Cox Proportional Hazard model, GAM: Generalized Additive Model, Cox-SPDE: Cox-Stochastic Partial Differential Equation Model, P-SPDE: Poisson-Stochastic Partial Differential Equation, RRb: Breeding site Relative Risk, Db: Breeding site Density, RRt: Treatment Relative Risk, Pop.Dens: Population Density, Risk0: Baseline Risk. (TIF 1247 kb)

Additional file 10: Figure S7. SR of the age effect with a baseline risk of 0.37. DGM: Data-Generating Model, Cox-PH: Cox Proportional Hazard model, GAM: Generalized Additive Model, Cox-SPDE: Cox-Stochastic Partial Differential Equation Model, P-SPDE: Poisson-Stochastic Partial Differential Equation, RRb: Breeding site Relative Risk, Db: Breeding site Density, RRt: Treatment Relative Risk, Pop.Dens: Population Density, Risk0: Baseline Risk. (TIF 1407 kb)

Additional file 11: Figure S8. Bias of the sex effect with a baseline risk of 0.37. DGM: Data-Generating Model, Cox-PH: Cox Proportional Hazard model, GAM: Generalized Additive Model, Cox-SPDE: Cox-Stochastic Partial Differential Equation Model, P-SPDE: Poisson-Stochastic Partial Differential Equation, RRb: Breeding site Relative Risk, Db: Breeding site Density, RRt: Treatment Relative Risk, Pop.Dens: Population Density, Risk0: Baseline Risk. (TIF 820 kb)

Additional file 12: Figure S9. MSE of the sex effect with a baseline risk of 0.37. DGM: Data-Generating Model, Cox-PH: Cox Proportional Hazard model, GAM: Generalized Additive Model, Cox-SPDE: Cox-Stochastic Partial Differential Equation Model, P-SPDE: Poisson-Stochastic Partial Differential Equation, RRb: Breeding site Relative Risk, Db: Breeding site Density, RRt: Treatment Relative Risk, Pop.Dens: Population Density, Risk0: Baseline Risk. (TIF 1147 kb)

Additional file 13: Figure S10. CR of the sex effect with a baseline risk of 0.37. DGM: Data-Generating Model, Cox-PH: Cox Proportional Hazard model, GAM: Generalized Additive Model, Cox-SPDE: Cox-Stochastic Partial Differential Equation Model, P-SPDE: Poisson-Stochastic Partial Differential Equation, RRb: Breeding site Relative Risk, Db: Breeding site Density, RRt: Treatment Relative Risk, Pop.Dens: Population Density, Risk0: Baseline Risk. (TIF $1380 \mathrm{~kb}$ )

Additional file 14: Figure S11. MSE of the treatment effect with the classic and bootstrap methods. DGM: Data-Generating Model, Cox-PH: Cox Proportional Hazard model, GAM: Generalized Additive Model, CoxSPDE: Cox-Stochastic Partial Differential Equation Model, P-SPDE: PoissonStochastic Partial Differential Equation, RRb: Breeding site Relative Risk, Db: Breeding site Density, RRt: Treatment Relative Risk, Pop.Dens: Population Density, RiskO: Baseline Risk. (TIF 650 kb)

\section{Abbreviations}

Cox-PH: Cox Proportional Hazard model; Cox-SPDE: Cox-Stochastic Partial Differential Equation; CR: Coverage Rate; GAM: Generalized Additive Model; HPP: Homogeneous Point Process; INLA: Integrated Nested Laplace Approximation; IPP: Inhomogeneous Point Process; MSE: Mean Square Error Pop.Dens: Population Density; P-SPDE: Poisson-Stochastic Partial Differential Equation; Risk0: Baseline Risk; SPDE: Stochastic Partial Differential Equation; SR: Significance Rate

\section{Acknowledgements}

We would like to thank Dr. Jean-Charles DUFOUR, administrator of the StatCalc server used to perform part of the simulations, as well as the Réseau Doctoral en Santé Publique, which is facilitated by the EHESP. Lastly, we are most grateful to Dr. Arianne Dorval for translating this article from French into English. The authors gratefully acknowledge Pr. Ogobara K. Doumbo, deceased in June 2018, for his invaluable scientific work and ideas.

\section{Authors' contributions}

AG, IS, and JG worked on the design of the simulation plan, the selection of models to compare, the interpretation of results, and the writing of the article. AG and $\mathrm{BO}$ contributed to the programming of the simulation with the $\mathrm{R}$ software and to the monitoring of intensive calculations on machines.

$I S, M H A, P D$, and $S H$ contributed to the implementation of the vaccine trial and to the collection and interpretation of data. $\mathrm{KS}, \mathrm{OD}, \mathrm{AD}$, and RG brought significant elements to the study at different stages. All authors participated in the review and validation of the final version of the article.

\section{Funding}

This work was funded by the French Development Agency and the "Prospective et Cooperation" association. Simulations were performed with the HPC resources of Aix-Marseille University (Mésocentre), which are funded as part of the Equip@Meso project (ANR-10-EQPX-29-01) of the "Investissements d'Avenir" program supervised by the French National Research Agency.

The dataset used for the application example was extracted from Vaccine Trial \#NCT02334462 (ClinicalTrials.gov registry), funded by the Laboratory of Malaria Immunology and Vaccinology (LMIV), National Institute of Allergy and Infectious Diseases (NIAID), National Institutes of Health (NIH), USA, and the Rodolphe Mérieux laboratory, Bamako, Mali. Issaka Sagara, Sara Healy, Ogobara K Doumbo and Patrick Duffy designed and wrote the protocol of this clinical trial.

None of these funding agencies or associations participated in the design of the study, in the collection, analysis, and interpretation of the data, or in the writing of the manuscript.

\section{Availability of data and materials}

The dataset used and/or analyzed in the present study is available from the corresponding author on reasonable request.

\section{Ethics approval and consent to participate}

Ethical clearance for Vaccine Trial \#NCT02334462 (ClinicalTrial.gov registry) was obtained from the ethics committee of the Faculty of Medecine and Denstry \& Faculty of pharmacy, Bamako, Mali (letter No2015_16_FMPOS, February 12 2015). Individual written consent and authorization were obtained from all participants.

\section{Consent for publication}

Not applicable.

\section{Competing interests}

Competing interests are related to Vaccine Trial \#NCT02334462 (ClinicalTrials. gov registry). The Rodolphe Mérieux laboratory was the manufacturer of the vaccine. Professor Patrick Duffy, co-author of this article, was appointed by the US National Institute of Health $(\mathrm{NIH})$, which also sponsored the vaccine trial.

\section{Author details}

${ }^{1}$ Aix Marseille Univ, INSERM, IRD, SESSTIM, Sciences Economiques \& Sociales de la Santé \& Traitement de l'Information Médicale, Marseille, France. ${ }^{2}$ Malaria Research and Training Center - Ogobara K Doumbo, FMOS-FAPH, Mali-NIAID-ICER, Université des Sciences, des Techniques et des Technologies de Bamako, Bamako, Mali. ${ }^{3}$ Direction des systèmes d'information en santé, Ministère de la santé, Ouagadougou, Burkina Faso. ${ }^{4}$ AP-HP, Hôpital Bichat, Unité de Recherche Clinique PNVS, Paris, France. ${ }^{5}$ Laboratory of Malaria Immunology and Vaccinology, National Institute of Allergy and Infectious Diseases, National Institutes of Health, Rockville, Maryland, USA. ${ }^{6}$ Aix Marseille Univ, APHM, INSERM, IRD, SESSTIM, Hop Timone, BioSTIC, Biostatistic \& ICT, Marseille, France.

Received: 26 November 2018 Accepted: 21 May 2019

Published online: 15 July 2019

\section{References}

1. Hiscox A, Homan T, Vreugdenhil C, Otieno B, Kibet A, Mweresa CK, et al. Spatial heterogeneity of malaria vectors and malaria transmission risk estimated using odour-baited mosquito traps. Malar J. 2014;13 Suppl 1:P41. doi:10.1186/1475-2875-13-S1-P41.

2. Tine RCK, Ndour CT, Faye B, Cairns M, Sylla K, Ndiaye M, et al. Feasibility, safety and effectiveness of combining home based malaria management and seasonal malaria chemoprevention in children less than 10 years in Senegal: a cluster-randomised trial. Trans R Soc Trop Med Hyg. 2014;108:1321. https://doi.org/10.1093/trstmh/trt103. 
3. Barry A, Issiaka D, Traore T, Mahamar A, Diarra B, Sagara I, et al. Optimal mode for delivery of seasonal malaria chemoprevention in Ouelessebougou. Mali: A cluster randomized trial. PLOS ONE. 2018;13: e0193296. https://doi.org/10.1371/journal.pone.0193296.

4. Tine RC, Faye B, Ndour CT, Ndiaye $J$, Ndiaye M, Bassene C, et al. Impact of combining intermittent preventive treatment with home management of malaria in children less than 10 years in a rural area of Senegal: a cluster randomized trial. Malar J. 2011;10:358. https://doi. org/10.1186/1475-2875-10-358.

5. Thera MA, Kone AK, Tangara B, Diarra E, Niare S, Dembele A, et al. School-aged children based seasonal malaria chemoprevention using artesunate-amodiaquine in Mali. Parasite Epidemiol Control. 2018;3:96105. https://doi.org/10.1016/j.parepi.2018.02.001.

6. Getachew Y, Janssen P, Yewhalaw D, Speybroeck N, Duchateau L. Coping with time and space in modelling malaria incidence: a comparison of survival and count regression models. Stat Med. 2013; 32:3224-33. https://doi.org/10.1002/sim.5752.

7. Li Y, Ryan L. Modeling spatial survival data using semiparametric frailty models. Biometrics. 2002;58:287-97. https://doi.org/10.1111/j. 0006-341X.2002.00287.x.

8. Gangnon RE, Clayton MK. A hierarchical model for spatially clustered disease rates. Stat Med. 2003;22:3213-28. https://doi.org/10.1002/sim.1570.

9. Sissoko MS, Sauerwein RW, Knight P, Bousema T, Coulibaly M, Samake $Y$, et al. Spatial patterns of Plasmodium falciparum clinical incidence, asymptomatic parasite carriage and Anopheles Density in Two Villages in Mali. Am J Trop Med Hyg. 2015;93:790-7. https://doi.org/ 10.4269/ajtmh.14-0765.

10. Overgaard HJ, Olano VA, Jaramillo JF, Matiz MI, Sarmiento D, Stenström TA, et al. A cross-sectional survey of Aedes aegypti immature abundance in urban and rural household containers in central Colombia. Parasit Vectors. 2017;10. https://doi.org/10.1186/ s13071-017-2295-1.

11. Imbahale SS, Paaijmans KP, Mukabana WR, van Lammeren R, Githeko AK, Takken W. A longitudinal study on Anopheles mosquito larval abundance in distinct geographical and environmental settings in western Kenya. Malar J. 2011;10. https://doi.org/10.1186/1475-2875-10-81.

12. Gao Q, Wang F, Lv X, Cao H, Su F, Zhou J, et al. Aedes albopictus production in urban stormwater catch basins and manhole chambers of downtown Shanghai. China. PLOS ONE. 2018;13:e0201607. https://doi.org/ 10.1371/journal.pone.0201607.

13. Pandey S, Das MK, Dhiman RC. Diversity of breeding habitats of anophelines (Diptera: Culicidae) in Ramgarh district, Jharkhand. India. J Vector Borne Dis. 2016:53-4:327-34.

14. Thomas S, Ravishankaran S, Justin JA, Asokan A, Mathai MT, Valecha N, et al. Overhead tank is the potential breeding habitat of Anopheles stephensi in an urban transmission setting of Chennai. India. Malar J. 2016;15. https://doi. org/10.1186/s12936-016-1321-7

15. Young RL, Weinberg J, Vieira V, Ozonoff A, Webster TF. A power comparison of generalized additive models and the spatial scan statistic in a casecontrol setting. Int J Health Geogr. 2010;9:37. https://doi.org/10.1186/1476072X-9-37.

16. Vieira VM, Weinberg JM, Webster TF. Individual-level space-time analyses of emergency department data using generalized additive modeling. BMC Public Health. 2012;12. https://doi.org/10.1186/1471-2458-12-687.

17. Rebaudet S, Griffiths K, Trazillio M, Lebeau A-G, Abedi AA, Bulit G, et al. Cholera spatial-temporal patterns in Gonaives, Haiti: From contributing factors to targeted recommendations. Adv Water Resour. 2017;108:377-85. https://doi.org/10.1016/j.advwatres.2016.12.012.

18. Tobler WR. A computer movie simulating urban growth in the detroit region. Econ Geogr. 1970;46:234. https://doi.org/10.2307/143141.

19. Lindgren F, Rue H, Lindström J. An explicit link between Gaussian fields and Gaussian Markov random fields: the stochastic partial differential equation approach. J R Stat Soc Ser B Stat Methodol. 2011;73:423-98. https://doi.org/10.1111/j.1467-9868.2011.00777.x.

20. Cameletti M, Lindgren F, Simpson D, Rue H. Spatio-temporal modeling of particulate matter concentration through the SPDE approach. AStA Adv Stat Anal. 2013;97:109-31. https://doi.org/10.1007/ s10182-012-0196-3.

21. Musenge E, Chirwa TF, Kahn K, Vounatsou P. Bayesian analysis of zero inflated spatiotemporal HIV/TB child mortality data through the INLA and SPDE approaches: Applied to data observed between 1992 and 2010 in rural North East South Africa. Int J Appl Earth Obs Geoinformation. 2013;22: 86-98. https://doi.org/10.1016/j.jag.2012.04.001.

22. Núñez O, Fernández-Navarro P, Martín-Méndez I, Bel-Lan A, Locutura JF, López-Abente G. Arsenic and chromium topsoil levels and cancer mortality in Spain. Environ Sci Pollut Res. 2016;23:17664-75. https://doi.org/10.1007/ s11356-016-6806-y.

23. Lenaerts E, Mandro M, Mukendi D, Suykerbuyk P, Dolo H, Wonya'Rossi $D$, et al. High prevalence of epilepsy in onchocerciasis endemic health areas in Democratic Republic of the Congo. Infect Dis Poverty. 2018;7. https://doi. org/10.1186/s40249-018-0452-1.

24. Burton A, Altman DG, Royston P, Holder RL. The design of simulation studies in medical statistics. Stat Med. 2006;25:4279-92. https://doi.org/10. 1002/sim.2673.

25. USAID. The DHS program STATcompiler. 2016. https://www.statcompiler. com/fr/. Accessed 1 Jan 2017.

26. Midega JT, Mbogo CM, Mwambi H, Wilson MD, Ojwang G, Mwangangi JM, et al. Estimating dispersal and survival of Anopheles gambiae and Anopheles funestus along the Kenyan coast by using mark-release-recapture methods. J Med Entomol. 2007:44:923-929. https://doi.org/10.1603/0022-2585(2007)44[923,EDASOA]2.0.CO;2. Accessed 24 Jul 2017.

27. Bousema T, Stresman G, Baidjoe AY, Bradley J, Knight P, Stone W, et al. The impact of hotspot-targeted interventions on malaria transmission in Rachuonyo south district in the western Kenyan highlands: A cluster-randomized controlled trial. PLOS Med. 2016;13: e1001993. https://doi.org/10.1371/journal.pmed.1001993.

28. Toure OA, Valecha N, Tshefu AK, Thompson R, Krudsood S, Gaye O, et al. A phase 3, double-blind, randomized study of arterolane maleate-piperaquine phosphate vs artemether-lumefantrine for falciparum malaria in adolescent and adult patients in Asia and Africa. Clin Infect Dis. 2016;62:964-71. https:// doi.org/10.1093/cid/ciw029.

29. Thera MA, Coulibaly D, Kone AK, Guindo AB, Traore K, Sall AH, et al. Phase 1 randomized controlled trial to evaluate the safety and immunogenicity of recombinant Pichia pastoris-expressed Plasmodium falciparum apical membrane antigen 1 (PfAMA1-FVO [25-545]) in healthy Malian adults in Bandiagara. Malar J. 2016;15. https://doi.org/10.1186/s12936-016-1466-4.

30. Sissoko MS, Healy SA, Katile A, Omaswa F, Zaidi I, Gabriel EE, et al. Safety and efficacy of PFSPZ Vaccine against Plasmodium falciparum via direct venous inoculation in healthy malaria-exposed adults in Mali: a randomised, double-blind phase 1 trial. Lancet Infect Dis. 2017;17:498-509. https://doi. org/10.1016/S1473-3099(17)30104-4.

31. Dama S, Niangaly H, Djimde M, Sagara I, Guindo CO, Zeguime A, et al. A randomized trial of dihydroartemisinin-piperaquine versus artemether-lumefantrine for treatment of uncomplicated Plasmodium falciparum malaria in Mali. Malar J. 2018;17. https://doi.org/10.1186/ s12936-018-2496-X.

32. Dicko A, Brown JM, Diawara H, Baber I, Mahamar A, Soumare HM, et al. Primaquine to reduce transmission of Plasmodium falciparum malaria in Mali: a single-blind, dose-ranging, adaptive randomised phase 2 trial. Lancet Infect Dis. 2016;16:674-84. https://doi.org/10.1016/S14733099(15)00479-X

33. Demographie M. Atlas des populations et pays du monde. 2017. https:// www.populationdata.net/pays/mali/. Accessed 1 Mar 2017.

34. Lee ET, Wang JW. Statistical methods for survival data analysis. 3rd ed. New York: J. Wiley; 2003. p. 1-165.

35. David C. Modelling survival data. In: Modelling survival data in medical research. 2nd ed. London: Chapman and Hall/CRC; 2003. p. 55-109.

36. Wood SN. Generalized additive models: an introduction with R. 2nd ed. London: Chapman and Hall/CRC; 2017. p. 136-71.

37. Rue H, Martino S, Chopin N. Approximate bayesian inference for latent gaussian models by using integrated nested Laplace approximations. J R Stat Soc Ser B Stat Methodol. 2009;71:319-92. https://doi.org/10.1111/j.14679868.2008.00700.x

38. Rue H, Riebler A, Sørbye SH, Illian JB, Simpson DP, Lindgren FK. Bayesian computing with INLA: a Review. Annu Rev Stat Its Appl. 2017:4:395-421. https://doi.org/10.1146/annurev-statistics-060116-054045.

39. Li L, Wu J, Wilhelm M, Ritz B. Use of generalized additive models and cokriging of spatial residuals to improve land-use regression estimates of nitrogen oxides in Southern California. Atmos Environ. 2012;55: 220-8. https://doi.org/10.1016/j.atmosenv.2012.03.035. 
40. Venables WN, Dichmont CM. GLMs, GAMs and GLMMs: an overview of theory for applications in fisheries research. Fish Res. 2004;70:319-37. https://doi.org/10.1016/j.fishres.2004.08.011.

41. Lindgren F, Rue H. Bayesian spatial modelling with R-INLA. J Stat Softw. 2015;63. doi:10.18637/jss.v063.i19.

42. Minasny B. McBratney AlexB. The Matèrn function as a general model for soil variograms. Geoderma. 2005;128:192-207. https://doi.org/10.1016/j. geoderma.2005.04.003.

43. Crowther MJ, Riley RD, Staessen JA, Wang J, Gueyffier F, Lambert PC. Individual patient data meta-analysis of survival data using Poisson regression models. BMC Med Res Methodol. 2012;12:34.

44. Simpson D, Illian JB, Lindgren F, Sørbye SH, Rue H. Going off grid: computationally efficient inference for log-Gaussian Cox processes. Biometrika. 2016;103:49-70. https://doi.org/10.1093/biomet/asv064.

45. Morris TP, White IR, Crowther MJ. Using simulation studies to evaluate statistical methods. Stat Med. 2019;38:2074-102. https://doi.org/10.1002/sim.8086.

46. Farrance CE, Rhee A, Jones RM, Musiychuk K, Shamloul M, Sharma S, et al. A plant-produced Pfs230 vaccine candidate blocks transmission of Plasmodium falciparum. Clin Vaccine Immunol. 2011;18:1351-7. https://doi. org/10.1128/CVI.05105-11.

47. Patrick E D. Study of the Safety and Immunogenicity of Pfs230D1M-EPA/ Alhydrogel and Pfs25M-EPA/Alhydrogel, a Transmission Blocking Vaccine Against Plasmodium Falciparum Malaria, in Adults in the U.S. and Mali. 2015 https://clinicaltrials.gov/ct2/show/study/NCT02334462?term=Pfs230\&cond= Malaria\%2CFalciparum\&cntry=ML. Accessed 5 Oct 2018.

48. Nissen A, Cook J, Loha E, Lindtiørn B. Proximity to vector breeding site and risk of Plasmodium vivax infection: a prospective cohort study in rural Ethiopia. Malar J. 2017;16. https://doi.org/10.1186/s12936-017-2031-5.

49. Umlauf N, Adler D, Kneib T, Lang S, Zeileis A. Structured additive regression models: An R interface to BayesX. J Stat Softw. 2015;63. doi:10.18637/jss.v063.i21.

50. Brezger A, Kneib T, Lang S. BayesX : Analyzing bayesian structured additive regression models. J Stat Softw. 2005;14. doi:10.18637/jss.v014.i11.

51. Adebayo SB, Gayawan E, Heumann C, Seiler C. Joint modeling of anaemia and malaria in children under five in Nigeria. Spat Spatio-Temporal Epidemiol. 2016;17:105-15. https://doi.org/10.1016/.jste.2016.04.011.

52. Fahrmeir $L$, Kneib T, Lang S. Penalized structured additive regression for space-time data: A bayesian perspective. Stat Sin. 2004;14:731-61 http:// www.jstor.org/stable/24307414. Accessed 20 March 2019.

53. Banerjee S, Wall MM, Carlin BP. Frailty modeling for spatially correlated survival data, with application to infant mortality in Minnesota. Biostatistics. 2003;4:123-42 https://academic.oup.com/biostatistics/article-abstract/4/1/ 123/246234. Accessed 24 Jul 2017.

54. Dekker FW, de Mutsert R, van Dijk PC, Zoccali C, Jager KJ. Survival analysis: time-dependent effects and time-varying risk factors. Kidney Int. 2008;74: 994-7. https://doi.org/10.1038/ki.2008.328.

55. Fisher LD, Lin DY. Time-dependent covariates in the cox proportionalhazards regression model. Annu Rev Public Health. 1999;20:145-57. https:// doi.org/10.1146/annurev publhealth.201.145.

56. Kenneth RH. Assessing time-by-covariate interactions in proportional hazards regression models using cubic spline functions. Sat Med. 1994; 13(10):1045-62 https://onlinelibrary.wiley.com/doi/pdf/10.1002/sim. 4780131007.

57. Wheeler DC, Waller LA, Cozen W, Ward MH. Spatial-temporal analysis of non-Hodgkin lymphoma risk using multiple residential locations. Spat Spatio-Temporal Epidemiol. 2012;3:163-71. https://doi.org/10.1016/.sste. 2012.04.009

\section{Publisher's Note}

Springer Nature remains neutral with regard to jurisdictional claims in published maps and institutional affiliations.

Ready to submit your research? Choose BMC and benefit from:

- fast, convenient online submission

- thorough peer review by experienced researchers in your field

- rapid publication on acceptance

- support for research data, including large and complex data types

- gold Open Access which fosters wider collaboration and increased citations

- maximum visibility for your research: over $100 \mathrm{M}$ website views per year

At BMC, research is always in progress.

Learn more biomedcentral.com/submissions 